\title{
Reducing the $\mathrm{O}(3)$ model as an effective field theory
}

\author{
Sven Bjarke Gudnason ${ }^{a}$ and Muneto Nitta ${ }^{b}$ \\ ${ }^{a}$ Institute of Contemporary Mathematics, School of Mathematics and Statistics, Henan University, \\ Kaifeng, Henan 475004, P.R. China \\ ${ }^{b}$ Department of Physics, and Research and Education Center for Natural Sciences, Keio University, \\ Hiyoshi 4-1-1, Yokohama, Kanagawa 223-8521, Japan \\ E-mail: gudnason@henu.edu.cn, nitta@phys-h.keio.ac.jp
}

ABSTRACT: We consider the $\mathrm{O}(3)$ or $\mathbb{C} P^{1}$ nonlinear sigma model as an effective field theory in a derivative expansion, with the most general Lagrangian that obeys $\mathrm{O}(3)$, parity and Lorentz symmetry. We work out the complete list of possible operators (terms) in the Lagrangian and eliminate as many as possible using integrations by parts. We further show at the four-derivative level, that the theory can be shown to avoid the Ostrogradsky instability, because the dependence on the d'Alembertian operator or so-called box, can be eliminated by a field redefinition. Going to the six-derivative order in the derivative expansion, we show that this can no longer be done, unless we are willing to sacrifice Lorentz invariance. By doing so, we can eliminate all dependence on double time derivatives and hence the Ostrogradsky instability or ghost, however, we unveil a remaining dynamical instability that takes the form either as a spiral instability or a runaway instability and estimate the critical field norm, at which the instability sets off.

Keywords: Effective Field Theories, Sigma Models

ARXIV EPRINT: 2110.15038 


\section{Contents}

1 Introduction and summary 1

2 The sigma model $\quad 5$

2.1 Group invariants and building blocks 6

$\begin{array}{lll}2.2 & \text { The dimension-2 operators } & 7\end{array}$

2.3 The baby-Skyrme term 9

2.4 Further identities 9

3 The sigma model as an EFT $\quad 10$

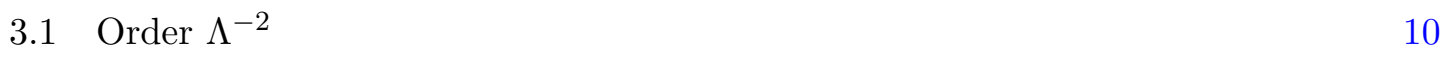

$\begin{array}{lll}3.2 & \text { Order } \Lambda^{-4} & 14\end{array}$

$\begin{array}{lr}\text { A Ostrogradsky's theorem } & \mathbf{3 4}\end{array}$

$\begin{array}{lll}\text { A.1 Example } & 35\end{array}$

B Field redefinition operators $\quad 35$

$\begin{array}{ll}\text { C Total derivatives } & 36\end{array}$

\section{Introduction and summary}

Effective field theories (EFTs) at low energies are very useful tools in classical and quantum field theories for formulating the most important impact of the full theory in terms of the low-energy variables (fields) valid at low energies up to the scale of the lightest field that has been integrated out, see e.g. ref. [1] for a review. Nowadays, it is even a commonly used approach for parametrizing our ignorance of new physics beyond the standard model and in that context it is called the standard model effective field theory (SMEFT) [2].

A more traditional low-energy EFT is the chiral Lagrangian as the low-energy theory of the strong interactions, which is a step more radical than the SMEFT, since the original fields, viz. quark and gluon fields, of quantum chromodynamics (QCD) are absent in the chiral Lagrangian theory and the lightest fields are the Nambu-Goldstone bosons of chiral symmetry breaking, namely the pions [3-5]. In chiral perturbation theory, the pions and other vector mesons are coupled to a nucleon field and the expansion is made order-byorder in the so-called chiral order which counts the number of derivatives or powers of quark masses, where a quark mass counts as two derivatives.

The leading-order term in the chiral Lagrangian, in the mesonic sector, is the kinetic term of the pions, which is written as a kinetic term of an $\mathrm{SU}(2)$-valued field containing three pions and an auxiliary field, traditionally called $\sigma$. The latter auxiliary field allows for a nonlinear constraint on the $\mathrm{SU}(2)$-valued field of the chiral Lagrangian, such that its 
determinant is manifestly equal to unity. This constraint in turn induces a nonvanishing curvature for the metric on the target space of the pions. For historic reasons and due to the choice of $\sigma$ as the symbol for the auxiliary field, such kind of theory, has henceforth been coined a nonlinear sigma model (NL $\sigma \mathrm{M})$.

Due to a low-energy theorem established by Weinberg [6], the effective field theory can be considered as a meaningful theory, despite the fact that it is nonrenormalizable and the systematic scheme usually utilized works by fixing a chiral order, $D$, to which calculations are carried out, and only considering $L \leq \frac{D}{2}-1$ loops in the theory.

A problem, however, naturally occurs at higher orders in a derivative expansion, namely that there may be more than one derivative acting on the same field. The simplest example with Lorentz invariance, is the square of the d'Alembertian operator on a field, $(\square \phi)^{2}$, which due to the theorem of Ostrogradsky [7], see also ref. [8], must have a linear dependence on at least one conjugate momentum, since the Hamiltonian corresponding to this Lagrangian contains $\left(\partial_{t}^{2} \phi\right)^{2}$. This readily implies that the energy is unbounded from below and from above and in turn that the theory is plagued by at least one ghost field. The necessary assumption for the Ostrogradsky theorem to hold, is that the Lagrangian is nondegenerate in the double time derivative, i.e. $\frac{\partial^{2} \mathcal{L}}{\left(\partial \partial_{t}^{2} \phi\right)^{2}} \neq 0$.

It is, however, well known that a fundamental theory can be absolutely sane, whereas upon writing down its effective low-energy theory, for example by integrating out a massive field, the resulting EFT turns out to be either nonlocal or in general plagued by the Ostrogradsky ghost, see e.g. ref. [9, section II.B]. It can further be argued that the Ostrogradsky ghost is naturally suppressed by the EFT energy scale, which is the energy scale of the lightest massive particle that has been integrated out, and hence can only be dynamically excited at energies of order of the EFT scale, which is by definition the energy scale where the EFT breaks down [9]. In case the theory at hand is in a certain class of asymptotically free theories, it has further been argued to have an effective mass that runs to infinity in the ultraviolet limit [10]. It is thus clear that the Ostrogradsky ghost, in a physically sane theory, should be considered as an artifact of the EFT and not a physically viable excitation (solution). Since the ghost by its nature comes with a kinetic term of the wrong sign, it furthermore implies the loss of unitarity [8], which is unfortunate for a quantum theory.

A well-known condition on a theory with higher derivatives, is to fine tune the coefficients of the higher-order derivative terms in such a way that the Euler-Lagrange equation of motion is of second order. This is indeed the way the Ostrogradsky ghost or instability is avoided in Galileon [11] and Horndeski [12] theories of gravity, the Skyrme model [13] (see ref. [14] for a higher order generalization), the Faddeev-Skyrme model [15], and the baby-Skyrme model $[16,17]$. This is of course like cherry-picking the theory in theory space and cannot be considered as the most general case.

One could contemplate ways to cure the theory in general, for instance by inventing certain projections that eliminate the unwanted features of the higher-derivative theory. One such approach has been carried out in Lee-Wick theory yielding a consistent unitary and renormalizable higher-derivative theory, however, at the cost of loss of causality at short distances [18]. It is claimed that the loss of microcausality does not propagate to larger length scales $[19,20]$. 
An Ostrogradsky ghost also exists in supersymmetric field theories with higher-dimensional operators in general $[21,22]$. One may eliminate the Ostrogradsky ghost by introducing an auxiliary gauge field to gauge it away, which was carried out explicitly in a supersymmetric chiral model [23] and in supergravity [24].

It has also been argued that the classical instability of the Ostrogradsky Hamiltonian does not pose as severe a problem in quantum theory as in classical physics [25], although the analysis performed here seems to require that the theory can be factorized, in terms of the partition function, into sane theories with some factors of the partition function being time reversed. In this case, it can be argued that the quantum field theory is not suffering directly from the Ostrogradsky instability, although microcausality is again certainly lost.

Yet another proposal is to restrict the theory to possess a certain reflection positivity, which essentially is a condition on the kinetic matrix of an equivalent theory made using more auxiliary fields, all with a positive sign of their respective kinetic term [26].

Nevertheless, it would be pleasant if it would be possible, already at the level of the Lagrangian, to eliminate the spurious or non-physical part of the theory, for instance, by means of finding an appropriate field redefinition (see e.g. ref. [1, section 6]) that can absorb the spurious part of the theory [9]. This is indeed possible [9] in simple theories, like the Ostrogradsky extension of the harmonic oscillator [8] and in Galileon modifications of gravity [11] up to mass dimension 11 [9]. Field redefinitions also play an important role in the renormalization of effective gauge field theories, see e.g. [27-29].

An important class of effective field theories is based on the $\mathrm{NL} \sigma \mathrm{M}$ with the addition of higher derivatives, the Skyrme model [13], the Faddeev-Skyrme model [15], and the baby-Skyrme model [16, 17], are examples. For solitons, like the Skyrmion or the FaddeevSkyrme knot, the higher-derivative term is a loophole in Derrick's theorem [30], preventing the soliton from collapsing. The latter two models are based on NL $\sigma \mathrm{Ms}$ with target space $S^{2}$ or $\mathbb{C} P^{1}$, whereas the first has target $S^{3} \sim \mathrm{SU}(2)$.

In this paper, we consider the $\mathrm{O}(3) \mathrm{NL} \sigma \mathrm{M}$ as an effective theory and write down the most general higher-order derivative operators and the goal is to simplify them as much as possible by integration-by-parts and by using field redefinitions. ${ }^{1}$ We will assume intact $\mathrm{O}(3)$ symmetry, parity symmetry and Lorentz (Poincaré) symmetry, and only relax the latter symmetry requirement at the end. The $\mathrm{O}(3) \mathrm{NL} \sigma \mathrm{M}$ is equivalent to the $\mathbb{C} P^{1} \mathrm{NL} \sigma \mathrm{M}$, by changing coordinates from a real vector field $\mathbf{n}: \mathbb{R}^{d+1} \rightarrow \mathbb{R}^{3}$ to a complex field $z: \mathbb{R}^{d+1} \rightarrow$ $\mathbb{C}$, which is the ordinary Riemann sphere coordinate. We will consider the model order-byorder in a derivative expansion, where each order is suppressed by further powers of a single intrinsic mass scale of the NL $\sigma \mathrm{M}$, taken as the scale up to which the EFT is trustable.

Our main motivation is two-fold. We would like to see whether it is possible to get rid of the Ostrogradsky instability in the $\mathrm{O}(3) \mathrm{NL} \sigma \mathrm{M}$ by simplifying and using field redefinitions, since the Ostrogradsky ghost and corresponding instability is non-physical and should be considered an artifact of the low-energy effective field theory. This is known not to be

\footnotetext{
${ }^{1}$ Similar considerations have already been made in the context of chiral perturbation theory to fourth [31], sixth [32], eighth [33] and even higher order [34] in derivatives. The analysis there is somewhat different, due to the phenomenological theory (chiral perturbation theory) being based on $\mathrm{SU}(2) \times \mathrm{SU}(2)$ symmetry and the fact that external gauge and scalar fields are included in the expansion, complicating the analysis.
} 
possible in other theories, like the chiral Lagrangian, but the only glimmer of hope here could be due integrability of the $\mathrm{O}(3) \mathrm{NL} \sigma \mathrm{M}$. The other motivation is to find a minimal formulation of the model, with an explicit and natural basis of operators describing the model. It is important in this regard that we clearly define what symmetries, continuous and discrete, are imposed on the theory for the result, since changing this assumption will allow further (or fewer) operators in the theory.

First we contemplate how to construct the most general Lagrangian of the $\mathrm{O}(3) \mathrm{NL} \sigma \mathrm{M}$ with unbroken $\mathrm{O}(3)$, parity and Lorentz symmetry. We establish in Lemma 1 that with the given symmetry assumptions, the number of derivatives must be even and hence so does the suppressing powers of the EFT scale, $\Lambda$. At the leading order in the derivative expansion, we establish the unique Lagrangian, up to an overall constant, in Theorem 2, Lemma 4 and Corollary 5.

At the next-to-leading order in the derivative expansion, which we denote $\Lambda^{-2}$, we exhaust the possibilities of terms or operators compatible with the symmetry requirements and show in Theorem 6, that the most general $\mathrm{O}(3) \mathrm{NL} \sigma \mathrm{M}$ to this order is box-free or d'Alembertian-free, viz. it contains no more than a single derivative operator acting on a field. This means that the most general theory - to this order - is described by a secondorder Euler-Lagrange equation of motion and is free from the Ostrogradsky instability or ghost. We utilize integrations by parts and field redefinitions to establish this result.

At the next-to-next-to-leading order in the derivative expansion, which we denote $\Lambda^{-4}$, we first write down the complete list of terms or operators to this order, namely containing six derivative operators and any number of fields (which can easily be shown to be 6,4 or 2 ). We then establish in Lemma 9, that the list of operators can be reduced by integrations by parts to a representative of 10 operators. We take into account the repercussions of the field redefinition used at order $\Lambda^{-2}$ to eliminate boxes or d'Alembertian operators, which generates a flurry of terms at the subsequent order, i.e. $\Lambda^{-4}$. Although we aim at removing all terms with more than one derivative acting on a field, we find that this does not seem possible. Therefore, we propose in Proposition 11 a field redefinition that simplifies the most general theory as much as possible, in the sense that only 4 operators with more than one derivative acting on a field remain, and all the terms induced by the field redefinition at order $\Lambda^{-2}$ have been eliminated. The theory still contains terms quadratic in double time derivatives acting on a field, but we show with Lemma 12, that they can be eliminated with a further field redefinition, leaving the theory with only linear dependence on double time derivatives on a field.

The linear dependence on double time derivatives, if the term is having a constant prefactor, is naturally canceled by the Legendre transform in going to the Hamiltonian. In fact, it is often considered as a sufficient condition for a higher-derivative theory to avoid the Ostrogradsky instability or ghost [35-40]; in fact, this works out for a single real field or for a real field theory where the prefactor of the double time derivative contains no dependence on single time derivatives of other fields and this is the content of Lemma 14. However, the conjugate momentum picks up new dependence on the double time derivative of the complex conjugated field at the linear level, which does not cancel out, and this is because the complex conjugate of the field is another field and hence the assumption of the Lemma fails and an Ostrogradsky-like instability is not avoided, as pointed out in Corollary 15. 
As a last resort, we choose to relax the assumption of intact Lorentz invariance, and make a frame dependent low-energy EFT suitable for the rest frame, which could be considered reasonable for a gapped theory at low energies. This is the proposal in Proposition 16, where a suitable field redefinition is found that eliminates all dependence on double time derivatives in the theory and hence the Ostrogradsky instability can manifestly be avoided. The reason why these linear terms in double time derivatives cannot be eliminated by field redefinitions when insisting on manifestly intact Lorentz symmetry, like the other problematic terms, is due to an incompatibility with the metric on $\mathbb{C} P^{1}$ of the form of said terms.

Although we have avoided the Ostrogradsky instability at order $\Lambda^{-4}$ in the derivative expansion of the theory, at the cost of sacrificing Lorentz invariance, we calculate the corresponding Hamiltonian of the theory in Lemma 20 and prove in Theorem 21 that the theory still suffers from either a spiral instability or a traditional runaway instability, that is turned on if the norm of the field reaches a critical value. Of course, this instability is simply due to the EFT breaking down at the scale $\Lambda$, but the mathematical nature of the spiral instability is different from the instability of the EFT breaking down at the previous $\left(\Lambda^{-2}\right)$ order in the derivative expansion.

The obvious extensions of our work that one could contemplate, are the extensions from $\mathrm{O}(3)$ to $\mathrm{O}(N)$ or to $\mathbb{C} P^{N-1}$, which we will leave as future work. In this direction, it may be interesting to see if it is easier (or harder) to eliminate higher-order derivative operators in the $\mathbb{C} P^{N-1}$ case, compared with the $\mathrm{O}(N)$ case, since the former enjoys a complex structure and as a target space manifold is Kähler. This work has repeatedly utilized integration by parts and discarded total derivatives, which is sensible on infinite flat Minkowski space $\mathbb{R}^{d+1}$, but it makes the analysis unsuitable for the theory if one wishes to apply it to condensed matter physics, like e.g. anti-ferromagnetism. In such case, one needs to keep track of every boundary term and analyze them one-by-one. We leave such a possibility for future studies.

\section{The sigma model}

The $\mathrm{O}(3)$ or $\mathbb{C} P^{1} \mathrm{NL} \sigma \mathrm{M}$ is given by

$$
\mathcal{L}=-\frac{1}{2} \partial_{\mu} \mathbf{n} \cdot \partial^{\mu} \mathbf{n}-\frac{\lambda}{2}(\mathbf{n} \cdot \mathbf{n}-1)
$$

where $\mathbf{n}=\left(n_{0}, n_{1}, n_{2}\right): \mathbb{R}^{d+1} \rightarrow S^{2}$ is a unit-length 3 -vector of real scalar field and $\lambda$ is a Lagrange multiplier imposing the unit-length or NL $\sigma \mathrm{M}$-constraint. The spacetime index $\mu=0,1, \ldots d$ runs over time and $d$-dimensional space and we are using the convention in which the flat Minkowski metric has the mostly-positive signature.

The equation of motion is given by

$$
\square \mathbf{n}=\lambda \mathbf{n},
$$

where $\square=\partial_{\mu} \partial^{\mu}$ is the d'Alembertian operator and by using the NL $\sigma \mathrm{M}$-constraint, $\lambda$ is given by $\mathbf{n} \cdot \square \mathbf{n}$. 
The coordinates $\mathbf{n}$ are called homogeneous coordinates. Another set of variables natural for the $\mathrm{O}(N)$ model are the inhomogeneous coordinates $\mathbf{m}=\left(m_{1}, m_{2}\right): \mathbb{R}^{d+1} \rightarrow \mathbb{R}^{2}$, which is an unconstrained 2-vector (or $(N-1)$-vector in the $\mathrm{O}(N)$ case), related to $\mathbf{n}$ as

$$
\mathbf{n}=\left(\frac{2 m_{1}}{1+\mathbf{m} \cdot \mathbf{m}}, \frac{2 m_{2}}{1+\mathbf{m} \cdot \mathbf{m}}, \frac{1-\mathbf{m} \cdot \mathbf{m}}{1+\mathbf{m} \cdot \mathbf{m}}\right),
$$

for which the Lagrangian (2.1) is given by

$$
\mathcal{L}=-2 \frac{\partial_{\mu} \mathbf{m} \cdot \partial^{\mu} \mathbf{m}}{(1+\mathbf{m} \cdot \mathbf{m})^{2}} .
$$

This generalizes to $\mathrm{O}(N)$ for any $N \geq 2$.

The above Lagrangian is exactly that of the $\mathbb{C} P^{1}$ model (for $N=3$ ) via the identification $\mathbb{C} \ni z=m_{1}+\mathrm{i} m_{2}$, for which the Lagrangian simply reads

$$
\mathcal{L}=-2 \frac{\partial_{\mu} z \partial^{\mu} \bar{z}}{\left(1+|z|^{2}\right)^{2}}
$$

clearly the map from $\mathbf{n}$ to $z$ is

$$
\mathbf{n}=\left(\frac{z+\bar{z}}{1+|z|^{2}},-\mathrm{i} \frac{z-\bar{z}}{1+|z|^{2}}, \frac{1-|z|^{2}}{1+|z|^{2}}\right),
$$

and $z$ is the Riemann (2-)sphere coordinate, which is the stereographic projection from $S^{2}$ to $\mathbb{C}$.

The advantage of working with inhomogeneous coordinates is that no constraints need to be taken into account, which will be crucial in the further analysis in this paper. Obviously, the Lagrangians (2.4) and (2.5) are identical, but offer straightforward generalizations to two different theories, namely the $\mathrm{O}(N) \mathrm{NL} \sigma \mathrm{M}$ and the $\mathbb{C} P^{N-1} \mathrm{NL} \sigma \mathrm{M}$, which is the reason for spelling them out here.

\subsection{Group invariants and building blocks}

Lemma 1 Suppose $\mathbf{n}$ is a unit-length 3-vector scalar field with mass-dimension 0, then all $\mathrm{O}(3)$ and Lorentz invariant terms must have an even number of derivatives.

Proof. All derivatives contracted by the inverse Minkowski metric come in pairs, so they must contribute an even number to the total number of derivatives, say $2 n$. In order to have an odd number of derivatives, we need a Lorentz invariant tensor structure with an odd number of spacetime indices. The only one is the Levi-Civita tensor

$$
\epsilon^{\mu_{0} \mu_{1} \cdots \mu_{d}}
$$

which has an odd number of spacetime indices when $d$ is even. Any combination of derivatives acting on a term that is contracted with the $\mathrm{O}(3)$ invariant $\delta_{a b}$ must vanish, due to the symmetry of the $\mathrm{O}(3)$ tensor structure versus the anti-symmetry of the Lorentz invariant Levi-Civita tensor. The only anti-symmetric $\mathrm{O}(3)$ invariant tensor is

$$
\epsilon_{a b c},
$$


so one might naively think that the following term is possible

$$
\epsilon^{\mu \nu \rho} \epsilon_{a b c} \partial_{\mu} n_{a} \partial_{\nu} n_{b} \partial_{\rho} n_{c}
$$

However, geometrically there are only two independent tangent vectors on $S^{2}$ and therefore if two derivatives correspond to orthogonal tangent vectors on $S^{2}$, the third must be a linear combination of the latter two and hence an anti-symmetric contraction must vanish. We have now ruled out any possible terms for $d=2$. For $d>2$, there are no anti-symmetric group structures of $\mathrm{O}(3)$ to contract with that can give a nonvanishing term. For $d=0$, there are no nonvanishing terms with one derivative. This completes the proof.

\subsection{The dimension-2 operators}

Theorem $\mathbf{2}$ Suppose $\mathbf{n}$ is a unit-length 3-vector scalar field with mass-dimension 0, then

$$
\partial_{\mu} \mathbf{n} \cdot \partial^{\mu} \mathbf{n},
$$

is the unique dimension 2 term with $\mathrm{O}(3)$ and Lorentz invariance in $d+1 \neq 2$ spacetime dimensions, whereas in $d+1=2$ spacetime dimensions there is additionally the topological term

$$
\epsilon^{\mu \nu} \mathbf{n} \cdot \partial_{\mu} \mathbf{n} \times \partial_{\nu} \mathbf{n}
$$

Proof. Using Lemma 1, no terms with a single derivative exist. The $\mathrm{O}(3)$-invariant tensor structures are $\delta_{a b}$ and $\epsilon_{a b c}$ and two derivatives must act on the term constructed with either tensor, since

$$
\begin{array}{r}
\mathbf{n} \cdot \partial_{\mu} \mathbf{n}=0, \\
\mathbf{n} \cdot \mathbf{n} \times \partial_{\mu} \mathbf{n}=0,
\end{array}
$$

vanish identically. The former is the NL $\sigma \mathrm{M}$ constraint and vanishes because

$$
\mathbf{n} \cdot \partial_{\mu} \mathbf{n}=\frac{1}{2} \partial_{\mu}(\mathbf{n} \cdot \mathbf{n})=0
$$

where $\mathbf{n} \cdot \mathbf{n}=1$ and the latter vanishes due to anti-symmetry of the $\mathrm{O}(3)$-invariant tensor and symmetry of the two n's. Hence, no composites can be made out of two $\mathrm{O}(3)$-invariants with a single spacetime derivative each.

Considering first the tensor $\delta_{a b}$, we can perform an integration by parts

$$
0=\partial_{\mu}\left(\mathbf{n} \cdot \partial^{\mu} \mathbf{n}\right)=\partial_{\mu} \mathbf{n} \cdot \partial^{\mu} \mathbf{n}+\mathbf{n} \cdot \square \mathbf{n}
$$

which vanishes identically due to the NL $\sigma \mathrm{M}$ constraint (2.12). Since the left-hand side of eq. (2.15) vanishes, $\mathbf{n} \cdot \square \mathbf{n}$ is equal to $-\partial_{\mu} \mathbf{n} \cdot \partial^{\mu} \mathbf{n}$ and there are no other ways of acting with two derivatives on the $\mathrm{O}(3)$ invariant $\delta_{a b}$.

Considering now the second tensor $\epsilon_{a b c}$, a single derivative vanishes due to eq. (2.13) and three anti-symmetrized derivatives vanish as well, see the proof of Lemma 1. The 
unique nonvanishing contraction with $\mathrm{O}(3)$ and Lorentz invariance, with dimension less than 4 , is thus

$$
\epsilon^{\mu \nu} \epsilon_{a b c} n_{a} \partial_{\mu} n_{b} \partial_{\nu} n_{c}=\epsilon^{\mu \nu} \mathbf{n} \cdot \partial_{\mu} \mathbf{n} \times \partial_{\nu} \mathbf{n},
$$

and hence the theorem follows.

Remark 3 The topological term (2.16) once integrated over spacetime, measures the topological degree of the mapping from (one-point compactified) $(1+1)$-dimensional spacetime (more precisely an Euclidean two-dimensional space after a Wick rotation) to $S^{2}$. More commonly used is the Lorentz vector

$$
Q^{\mu}=\epsilon^{\mu \nu \rho} \mathbf{n} \cdot \partial_{\nu} \mathbf{n} \times \partial_{\rho} \mathbf{n},
$$

whose time-component, when integrated over space, represents the static topological degree from (one-point compactified) 2-dimensional space to $S^{2}$. In some literature, this is known as the baby-Skyrme charge and in other it is known as the vortex charge or vorticity.

Lemma 4 The $\mathrm{O}(3)$ and Lorentz invariant term

$$
\frac{\mathrm{i} \epsilon^{\mu \nu} \partial_{\mu} z \partial_{\nu} \bar{z}}{\left(1+|z|^{2}\right)^{2}} F(z, \bar{z})
$$

does not contribute to the equations of motion. $F=\bar{F}$ is a real function of the field $z$ and its complex conjugate.

Proof. The Lagrangian

$$
\mathcal{L}=-\mathrm{i} \epsilon^{\mu \nu} \partial_{\mu} z \partial_{\nu} \bar{z} F(z, \bar{z})
$$

has the corresponding equation of motion for $\bar{z}$ :

$$
\begin{aligned}
0 & =\partial_{\nu}\left(\mathrm{i} \epsilon^{\mu \nu} \partial_{\mu} z F\right)-\mathrm{i} \epsilon^{\mu \nu} \partial_{\mu} z \partial_{\nu} \bar{z} \frac{\partial F}{\partial \bar{z}} \\
& =\mathrm{i} \epsilon^{\mu \nu} \partial_{\mu} \partial_{\nu} z F+\mathrm{i} \epsilon^{\mu \nu} \partial_{\mu} z \partial_{\nu} z \frac{\partial F}{\partial z}
\end{aligned}
$$

which is identically zero due to the antisymmetry of $\epsilon^{\mu \nu}$. Absorbing the metric factor into $F(z, \bar{z})$ completes the proof.

Corollary $\mathbf{5}$ Suppose $\mathbf{n}$ is a unit-length 3-vector scalar field with mass-dimension 0, then

$$
\partial_{\mu} \mathbf{n} \cdot \partial^{\mu} \mathbf{n}
$$

is the unique dimension 2 term with $\mathrm{O}(3)$ and Lorentz invariance that contributes to the equation of motion.

Proof. Using Theorem 2, the only other term than $\partial_{\mu} \mathbf{n} \cdot \partial^{\mu} \mathbf{n}$ is given by $\epsilon^{\mu \nu} \mathbf{n} \cdot \partial_{\mu} \mathbf{n} \times \partial_{\nu} \mathbf{n}$ and is a Lorentz invariant only in $d+1=2$ dimensions. The latter term is shown not to contribute to the equations of motion, by setting $F=1$ (or any constant) in Lemma 4 . 


\subsection{The baby-Skyrme term}

The baby-Skyrme term is a special dimension- 4 derivative operator, given by

$$
\left(\mathbf{n} \cdot \partial_{\mu} \mathbf{n} \times \partial_{\nu} \mathbf{n}\right)\left(\mathbf{n} \cdot \partial^{\mu} \mathbf{n} \times \partial^{\nu} \mathbf{n}\right) .
$$

By using the identity

$$
\epsilon_{a b c} \epsilon_{d e f}=\delta_{a d}\left(\delta_{b e} \delta_{c f}-\delta_{b f} \delta_{c e}\right)-\delta_{a e}\left(\delta_{b d} \delta_{c f}-\delta_{b f} \delta_{c d}\right)+\delta_{a f}\left(\delta_{b d} \delta_{c e}-\delta_{a e} \delta_{c d}\right),
$$

it is easy to see that only the first term is nonvanishing for the Skyrme term (2.22) due to the NL $\sigma \mathrm{M}$-constraint (2.12) and hence the Skyrme term obeys the identity

$$
\begin{aligned}
\left(\mathbf{n} \cdot \partial_{\mu} \mathbf{n} \times \partial_{\nu} \mathbf{n}\right)\left(\mathbf{n} \cdot \partial^{\mu} \mathbf{n} \times \partial^{\nu} \mathbf{n}\right) & =\left(\partial_{\mu} \mathbf{n} \times \partial_{\nu} \mathbf{n}\right) \cdot\left(\partial^{\mu} \mathbf{n} \times \partial^{\nu} \mathbf{n}\right) \\
& =\left(\partial_{\mu} \mathbf{n} \cdot \partial^{\mu} \mathbf{n}\right)^{2}-\left(\partial_{\mu} \mathbf{n} \cdot \partial_{\nu} \mathbf{n}\right)\left(\partial^{\mu} \mathbf{n} \cdot \partial^{\nu} \mathbf{n}\right) .
\end{aligned}
$$

It is thus clear that the Skyrme term equivalently can be viewed as the special combination of the two latter terms with relative coefficient 1 and -1 , respectively. Although the last term in the second line above has a negative coefficient, it is clear from the left-hand side that this specific combination is positive semi-definite on a Euclidean manifold.

It will prove convenient to rewrite the Skyrme term in inhomogeneous coordinates:

$$
8 \frac{\left(\partial_{\mu} z \partial^{\mu} \bar{z}\right)\left(\partial_{\nu} z \partial^{\nu} \bar{z}\right)-\left(\partial_{\mu} z \partial_{\nu} \bar{z}\right)\left(\partial^{\mu} z \partial^{\nu} \bar{z}\right)}{\left(1+|z|^{2}\right)^{4}} .
$$

\subsection{Further identities}

Let us note that acting with derivatives on the nonlinear sigma model constraint, $\mathbf{n} \cdot \mathbf{n}=1$, yields

$$
\begin{aligned}
\mathbf{n} \cdot \partial_{\mu} \mathbf{n} & =0 \\
\partial_{\mu} \mathbf{n} \cdot \partial_{\nu} \mathbf{n}+\mathbf{n} \cdot \partial_{\mu} \partial_{\nu} \mathbf{n} & =0 \\
\partial_{\mu} \mathbf{n} \cdot \partial_{\nu} \partial_{\rho} \mathbf{n}+\partial_{\nu} \mathbf{n} \cdot \partial_{\mu} \partial_{\rho} \mathbf{n}+\partial_{\rho} \mathbf{n} \cdot \partial_{\mu} \partial_{\nu} \mathbf{n}+\mathbf{n} \cdot \partial_{\mu} \partial_{\nu} \partial_{\rho} \mathbf{n} & =0
\end{aligned}
$$

where the first equation is the already well-used identity (2.12) and the following equations are generalizations thereof. The largest tensor structure we will be needing here is a spin-3 tensor (three free Lorentz indices), since the Lorentz-invariant operator that can be built from such a tensor must have mass dimension 6 or higher and that will be the largest mass dimension we will consider in this paper.

Contracting two free Lorentz indices with the inverse Minkowski metric in eqs. (2.27) and (2.28) yields

$$
\begin{array}{r}
\partial_{\mu} \mathbf{n} \cdot \partial^{\mu} \mathbf{n}+\mathbf{n} \cdot \square \mathbf{n}=0, \\
2 \partial_{\mu} \partial_{\rho} \mathbf{n} \cdot \partial_{\nu} \partial \rho \mathbf{n}+\square \mathbf{n} \cdot \partial_{\mu} \partial_{\nu} \mathbf{n}+\partial_{\mu} \mathbf{n} \cdot \square \partial_{\nu} \mathbf{n}+\partial_{\mu} \square \mathbf{n} \cdot \partial_{\nu} \partial_{\nu} \mathbf{n}+2 \partial_{\rho} \mathbf{n} \cdot \partial_{\mu} \partial_{\nu} \partial_{\rho} \mathbf{n}+\mathbf{n} \cdot \partial_{\mu} \partial_{\nu} \square \mathbf{n}=0,
\end{array}
$$

whereas the latter identity is obtained by acting with the d'Alembertian on eq. (2.27). 
The similar constraint with two contracted pairs of Lorentz indices is given by

$$
4 \partial_{\mu} \mathbf{n} \cdot \partial^{\mu} \square \mathbf{n}+2 \partial_{\mu} \partial_{\nu} \mathbf{n} \cdot \partial^{\mu} \partial^{\nu} \mathbf{n}+\square \mathbf{n} \cdot \square \mathbf{n}+\mathbf{n} \cdot \square^{2} \mathbf{n}=0
$$

which is obtained by acting with the d'Alembertian on eq. (2.29). Two Lorentz contractions yield a minimum mass dimension- 4 operator and the dimension- 5 operator would have one free Lorentz index, which for making at most dimension- 6 operators would vanish, since it can only be contracted with the term of eq. (2.26).

The final constraint contains three pairs of Lorentz-contracted indices

$$
\begin{aligned}
& 12 \partial_{\mu} \partial_{\nu} \mathbf{n} \cdot \partial^{\mu} \partial^{\nu} \square \mathbf{n}+6 \partial_{\mu} \square \mathbf{n} \cdot \partial^{\mu} \square \mathbf{n}+6 \partial_{\mu} \mathbf{n} \cdot \partial^{\mu} \square^{2} \mathbf{n} \\
& \quad+4 \partial_{\mu} \partial_{\nu} \partial_{\rho} \mathbf{n} \cdot \partial^{\mu} \partial^{\nu} \partial^{\rho} \mathbf{n}+3 \square \mathbf{n} \cdot \square^{2} \mathbf{n}+\mathbf{n} \cdot \square^{3} \mathbf{n}=0,
\end{aligned}
$$

which is found by acting with the d'Alembertian on eq. (2.32). For a Lorentz invariant operator with mass dimension 6 , this is the only identity.

\section{The sigma model as an EFT}

We will now consider the NL $\sigma \mathrm{M}$ as an EFT and make a derivative expansion, but conserving $\mathrm{O}(3)$ and Lorentz invariance. The program we will employ here is to use field redefinitions to eliminate as many derivative operators as possible. In order to set up the derivative expansion, we will assume that the $\mathrm{NL} \sigma \mathrm{M}$ only has one scale $\Lambda$ and hence up to an irrelevant overall constant factor, and using Corollary 5 , we have

$$
\begin{aligned}
\mathcal{L} & =-\frac{1}{2} \Lambda^{d-1}\left\{\partial_{\mu} \mathbf{n} \cdot \partial^{\mu} \mathbf{n}+\mathcal{O}\left(\Lambda^{-2}\right)+\lambda(\mathbf{n} \cdot \mathbf{n}-1)\right\} \\
& =-2 \Lambda^{d-1}\left\{\frac{\partial_{\mu} z \partial^{\mu} \bar{z}}{\left(1+|z|^{2}\right)^{2}}+\mathcal{O}\left(\Lambda^{-2}\right)\right\}
\end{aligned}
$$

for the theory in $(d+1)$-dimensional spacetime and the order $\Lambda^{-2}$ term represents fourthorder derivative terms, which thus have to be suppressed by a factor of $\Lambda^{2}$. Since we have assumed that there is only one energy scale in the theory, it must be proportional to $\Lambda$. $\lambda$ is a Lagrange multiplier enforcing the unit length of the 3-vector field $\mathbf{n}$ and we have conveniently written the model in both the vector $(\mathbf{n})$ and stereographic $(z)$ coordinates.

\subsection{Order $\Lambda^{-2}$}

We will now write down the most general $\mathrm{O}(3) \mathrm{NL} \sigma \mathrm{M}$ to order $\Lambda^{-2}$ in the EFT expansion. Recalling Lemma 1, there are no $\mathrm{O}(3)$ and Lorentz invariant terms with an odd number of derivatives and hence no terms of order $\Lambda^{-1}, \Lambda^{-3}, \cdots$ and so on. 
The complete list of dimension 4 operators is

$$
\begin{array}{r}
\left(\partial_{\mu} \mathbf{n} \cdot \partial^{\mu} \mathbf{n}\right)\left(\partial_{\nu} \mathbf{n} \cdot \partial^{\nu} \mathbf{n}\right), \\
\left(\partial_{\mu} \mathbf{n} \cdot \partial_{\nu} \mathbf{n}\right)\left(\partial^{\mu} \mathbf{n} \cdot \partial^{\nu} \mathbf{n}\right), \\
\mathbf{n} \cdot \square^{2} \mathbf{n}, \\
\partial_{\mu} \mathbf{n} \cdot \partial^{\mu} \square \mathbf{n}, \\
\square \mathbf{n} \cdot \square \mathbf{n}, \\
\partial_{\mu} \partial_{\nu} \mathbf{n} \cdot \partial^{\mu} \partial^{\nu} \mathbf{n}, \\
\left(\epsilon^{\mu \nu} \mathbf{n} \cdot \partial_{\mu} \mathbf{n} \times \partial_{\nu} \mathbf{n}\right)\left(\epsilon^{\rho \sigma} \mathbf{n} \cdot \partial_{\rho} \mathbf{n} \times \partial_{\sigma} \mathbf{n}\right), \\
\left(\epsilon^{\mu \nu} \mathbf{n} \cdot \partial_{\mu} \mathbf{n} \times \partial_{\nu} \mathbf{n}\right)\left(\partial_{\rho} \mathbf{n} \cdot \partial^{\rho} \mathbf{n}\right),
\end{array}
$$

where we have already eliminated possibilities that are related by the identities $(2.27)$ and (2.29) and the latter two operators (in eqs. (3.7) and (3.8)) are Lorentz invariant only in $d+1=2$ dimensions.

The identity (2.32) can be used to eliminate one of the four dimension-4 operators (3.4)-(3.7). However, integration by parts (IBP) and discarding total derivatives, relates the operators (3.4)-(3.7), which can easily be shown:

$$
\begin{aligned}
\partial_{\mu}\left(\mathbf{n} \cdot \partial^{\mu} \square \mathbf{n}\right) & =\partial_{\mu} \mathbf{n} \cdot \partial^{\mu} \square \mathbf{n}+\mathbf{n} \cdot \square^{2} \mathbf{n}, \\
\partial_{\mu}\left(\partial^{\mu} \mathbf{n} \cdot \square \mathbf{n}\right) & =\square \mathbf{n} \cdot \square \mathbf{n}+\partial_{\mu} \mathbf{n} \cdot \partial^{\mu} \square \mathbf{n}, \\
\partial_{\mu}\left(\partial_{\nu} \mathbf{n} \cdot \partial^{\mu} \partial^{\nu} \mathbf{n}\right) & =\partial_{\mu} \partial_{\nu} \mathbf{n} \cdot \partial^{\mu} \partial^{\nu} \mathbf{n}+\partial_{\mu} \mathbf{n} \cdot \partial^{\mu} \square \mathbf{n},
\end{aligned}
$$

yielding

$$
\begin{array}{r}
\left(\partial_{\mu} \mathbf{n} \cdot \partial^{\mu} \mathbf{n}\right)\left(\partial_{\nu} \mathbf{n} \cdot \partial^{\nu} \mathbf{n}\right), \\
\left(\partial_{\mu} \mathbf{n} \cdot \partial_{\nu} \mathbf{n}\right)\left(\partial^{\mu} \mathbf{n} \cdot \partial^{\nu} \mathbf{n}\right), \\
\square \mathbf{n} \cdot \square \mathbf{n},
\end{array}
$$

where the operator (3.8) has been eliminated from the above list due to its relation to the existing operators (3.2) and (3.3). First using the identity

$$
\epsilon^{\mu \nu} \epsilon^{\rho \sigma}=\delta^{\mu \rho} \delta^{\nu \sigma}-\delta^{\mu \sigma} \delta^{\nu \rho},
$$

we have

$$
\left(\epsilon^{\mu \nu} \mathbf{n} \cdot \partial_{\mu} \mathbf{n} \times \partial_{\nu} \mathbf{n}\right)\left(\epsilon^{\rho \sigma} \mathbf{n} \cdot \partial_{\rho} \mathbf{n} \times \partial_{\sigma} \mathbf{n}\right)=2\left(\mathbf{n} \cdot \partial_{\mu} \mathbf{n} \times \partial_{\nu} \mathbf{n}\right)\left(\mathbf{n} \cdot \partial^{\mu} \mathbf{n} \times \partial^{\nu} \mathbf{n}\right),
$$

and then using the identity (2.23), we have

$$
\left(\epsilon^{\mu \nu} \mathbf{n} \cdot \partial_{\mu} \mathbf{n} \times \partial_{\nu} \mathbf{n}\right)\left(\epsilon^{\rho \sigma} \mathbf{n} \cdot \partial_{\rho} \mathbf{n} \times \partial_{\sigma} \mathbf{n}\right)=2\left(\partial_{\mu} \mathbf{n} \cdot \partial^{\mu} \mathbf{n}\right)\left(\partial_{\nu} \mathbf{n} \cdot \partial^{\nu} \mathbf{n}\right)-2\left(\partial_{\mu} \mathbf{n} \cdot \partial_{\nu} \mathbf{n}\right)\left(\partial^{\mu} \mathbf{n} \cdot \partial^{\nu} \mathbf{n}\right)
$$

The operator (3.9), on the other hand, has been eliminated due to the following considerations. Consider a field configuration with a non-negative instanton density $\left(\epsilon^{\mu \nu} \mathbf{n}\right.$. $\left.\partial_{\mu} \mathbf{n} \times \partial_{\nu} \mathbf{n}\right) \geq 0$; for such a configuration, the operator (3.9) is bounded from below and 
perturbations do not destabilize the theory. Now consider a parity transformation of the latter configuration $\left(x^{0}, x^{1}\right) \rightarrow\left(x^{0},-x^{1}\right)$; this turns instantons into anti-instanton and anti-instantons into instantons. Now the operator (3.9), however, is non-positive and is bounded from above. This has the dire consequence that perturbations of such a field will destabilize the theory via a runaway instability. We have thus justified the assumption that the theory should better be parity invariant, hence eliminating the operator (3.9).

One may wonder if the constraint $(2.32)$ can be used to eliminate the last operator containing boxes, i.e. (3.15). Consider thus

$$
\left(b_{1}+b\right)\left(\mathbf{n} \cdot \square^{2} \mathbf{n}\right)+\left(b_{2}+4 b\right)\left(\partial_{\mu} \mathbf{n} \cdot \partial^{\mu} \square \mathbf{n}\right)+\left(b_{3}+2 b\right)\left(\partial_{\mu} \partial_{\nu} \mathbf{n} \cdot \partial^{\mu} \partial^{\nu} \mathbf{n}\right)+\left(b_{4}+b\right)(\square \mathbf{n} \cdot \square \mathbf{n}) .
$$

If we integrate the first three operators by parts, we get

$$
\begin{aligned}
& \left(b_{1}-b_{2}+b_{3}+b_{4}\right)(\square \mathbf{n} \cdot \square \mathbf{n})+\left(b_{1}+b\right) \partial_{\mu}\left(\mathbf{n} \cdot \partial^{\mu} \square \mathbf{n}\right) \\
& \quad+\left(b_{2}-b_{1}-b_{3}-b\right) \partial_{\mu}\left(\partial^{\mu} \mathbf{n} \cdot \square \mathbf{n}\right)+\left(b_{3}+2 b\right) \partial_{\mu}\left(\partial_{\nu} \mathbf{n} \cdot \partial^{\mu} \partial^{\nu} \mathbf{n}\right),
\end{aligned}
$$

where the terms with coefficient $b$ vanish due to the constraint (2.32). Unfortunately, the remaining (first) term in the above equation, which is not a total derivative, does not depend on $b$; therefore, no usage of adjusting $b$ can eliminate the last term. The complete list of dimension-4 operators with $\mathrm{O}(3)$, parity and Lorentz invariance is thus given by eqs. (3.13)-(3.15).

Theorem 6 The $N L \sigma M$ with $\mathrm{O}(3)$, parity and Lorentz invariance having a single mass scale $\Lambda$, written as

$$
\begin{aligned}
\mathcal{L}=-\frac{\Lambda^{d-1}}{2}\{ & \left(\partial_{\mu} \mathbf{n} \cdot \partial^{\mu} \mathbf{n}\right)+\frac{c_{4}+c_{4}^{\prime}}{\Lambda^{2}}\left(\partial_{\mu} \mathbf{n} \cdot \partial^{\mu} \mathbf{n}\right)\left(\partial_{\nu} \mathbf{n} \cdot \partial^{\nu} \mathbf{n}\right)-\frac{c_{4}}{\Lambda^{2}}\left(\partial_{\mu} \mathbf{n} \cdot \partial_{\nu} \mathbf{n}\right)\left(\partial^{\mu} \mathbf{n} \cdot \partial^{\nu} \mathbf{n}\right) \\
& \left.+\frac{c_{4} \square}{\Lambda^{2}}(\square \mathbf{n} \cdot \square \mathbf{n})+\mathcal{O}\left(\Lambda^{-4}\right)+\lambda(\mathbf{n} \cdot \mathbf{n}-1)\right\},
\end{aligned}
$$

subject to the constraints $c_{4}+c_{4}^{\prime} \geq 0, c_{4}^{\prime} \geq 0, c_{4 \square} \geq 0$ can, to order $\Lambda^{-2}$, be reduced to

$$
\begin{aligned}
\mathcal{L}=-\frac{\Lambda^{d-1}}{2}\{ & \left(\partial_{\mu} \mathbf{n} \cdot \partial^{\mu} \mathbf{n}\right)+\frac{c_{4}+c_{4}^{\prime}+c_{4} \square}{\Lambda^{2}}\left(\partial_{\mu} \mathbf{n} \cdot \partial^{\mu} \mathbf{n}\right)\left(\partial_{\nu} \mathbf{n} \cdot \partial^{\nu} \mathbf{n}\right) \\
& \left.-\frac{c_{4}}{\Lambda^{2}}\left(\partial_{\mu} \mathbf{n} \cdot \partial_{\nu} \mathbf{n}\right)\left(\partial^{\mu} \mathbf{n} \cdot \partial^{\nu} \mathbf{n}\right)+\mathcal{O}\left(\Lambda^{-4}\right)+\lambda(\mathbf{n} \cdot \mathbf{n}-1)\right\},
\end{aligned}
$$

via a field redefinition, where the latter formulation of the theory contains no d'Alembertian operators.

Proof. First we write the theory (3.21) in terms of the stereographic coordinate, $z$ :

$$
\begin{aligned}
\mathcal{L}=-2 \Lambda^{d-1}\{ & \frac{\partial_{\mu} z \partial^{\mu} \bar{z}}{\left(1+|z|^{2}\right)^{2}}+\frac{2 c_{4}+4 c_{4}^{\prime}+4 c_{4} \square}{\Lambda^{2}} \frac{\left(\partial_{\mu} z \partial^{\mu} \bar{z}\right)^{2}}{\left(1+|z|^{2}\right)^{4}}-\frac{2 c_{4}+4 c_{4 \square}}{\Lambda^{2}} \frac{\left(\partial_{\mu} z \partial^{\mu} z\right)\left(\partial_{\nu} \bar{z} \partial^{\nu} \bar{z}\right)}{\left(1+|z|^{2}\right)^{4}} \\
& +\frac{4 c_{4 \square}}{\Lambda^{2}} \frac{\left(\partial_{\mu} z \partial^{\mu} z\right)\left(\partial_{\nu} \bar{z} \partial^{\nu} \bar{z}\right)}{\left(1+|z|^{2}\right)^{3}}-\frac{2 c_{4} \square}{\Lambda^{2}} \frac{\left(\partial_{\mu} z \partial^{\mu} z\right)(\bar{z} \square \bar{z})}{\left(1+|z|^{2}\right)^{3}}-\frac{2 c_{4 \square}}{\Lambda^{2}} \frac{\left(\partial_{\mu} \bar{z} \partial^{\mu} \bar{z}\right)(z \square z)}{\left(1+|z|^{2}\right)^{3}} \\
& \left.+\frac{c_{4} \square}{\Lambda^{2}} \frac{\square z \square \bar{z}}{\left(1+|z|^{2}\right)^{2}}+\mathcal{O}\left(\Lambda^{-4}\right)\right\} .
\end{aligned}
$$


Now considering the following field redefinition

$$
z \rightarrow z+\frac{1}{\Lambda^{2}} \psi
$$

to order $\Lambda^{-2}$, the terms generated by $\psi$ can only come from the first term (the kinetic term) in the theory.

It is easily shown that

$$
\begin{aligned}
\frac{\partial_{\mu} z \partial^{\mu} \bar{z}}{\left(1+|z|^{2}\right)^{2}} \rightarrow & \frac{\partial_{\mu} z \partial^{\mu} \bar{z}}{\left(1+|z|^{2}\right)^{2}}+\frac{1}{\Lambda^{2}}\left[\frac{\partial_{\mu} \psi \partial^{\mu} \bar{z}}{\left(1+|z|^{2}\right)^{2}}+\frac{\partial_{\mu} z \partial^{\mu} \bar{\psi}}{\left(1+|z|^{2}\right)^{2}}-\frac{2\left(\partial_{\mu} z \partial^{\mu} \bar{z}\right)(z \bar{\psi}+\psi \bar{z})}{\left(1+|z|^{2}\right)^{3}}\right] \\
& +\mathcal{O}\left(\Lambda^{-4}\right) \\
= & \frac{\partial_{\mu} z \partial^{\mu} \bar{z}}{\left(1+|z|^{2}\right)^{2}} \\
& +\frac{1}{\Lambda^{2}}\left[-\frac{\square z \bar{\psi}}{\left(1+|z|^{2}\right)^{2}}-\frac{\square \bar{z} \psi}{\left(1+|z|^{2}\right)^{2}}+\frac{2\left(\partial_{\mu} z \partial^{\mu} z\right) \bar{z} \bar{\psi}}{\left(1+|z|^{2}\right)^{3}}+\frac{2\left(\partial_{\mu} \bar{z} \partial^{\mu} \bar{z}\right) z \psi}{\left(1+|z|^{2}\right)^{3}}\right] \\
& +\mathcal{O}\left(\Lambda^{-4}\right) .
\end{aligned}
$$

An educated guess is to take $\psi \propto \square z$, but unfortunately one can only remove either the term with two boxes or the two terms with one box; thus another term in $\psi$ is necessary. Choosing instead

$$
\psi=\alpha \square z+\gamma \frac{\left(\partial_{\mu} z \partial^{\mu} z\right) \bar{z}}{1+|z|^{2}},
$$

a straightforward calculation yields

$$
\begin{aligned}
\mathcal{L}=-2 \Lambda^{d-1}\{ & \frac{\partial_{\mu} z \partial^{\mu} \bar{z}}{\left(1+|z|^{2}\right)^{2}}+\frac{2 c_{4}+4 c_{4}^{\prime}+4 c_{4} \square}{\Lambda^{2}} \frac{\left(\partial_{\mu} z \partial^{\mu} \bar{z}\right)^{2}}{\left(1+|z|^{2}\right)^{4}} \\
& -\frac{2 c_{4}+4 c_{4 \square}+4 \gamma}{\Lambda^{2}} \frac{\left(\partial_{\mu} z \partial^{\mu} z\right)\left(\partial_{\nu} \bar{z} \partial^{\nu} \bar{z}\right)}{\left(1+|z|^{2}\right)^{4}}+\frac{4 c_{4 \square}+4 \gamma}{\Lambda^{2}} \frac{\left(\partial_{\mu} z \partial^{\mu} z\right)\left(\partial_{\nu} \bar{z} \partial^{\nu} \bar{z}\right)}{\left(1+|z|^{2}\right)^{3}} \\
& -\frac{2 c_{4 \square}+\gamma-2 \alpha}{\Lambda^{2}} \frac{\left(\partial_{\mu} z \partial^{\mu} z\right)(\bar{z} \square \bar{z})}{\left(1+|z|^{2}\right)^{3}}-\frac{2 c_{4 \square}+\gamma-2 \alpha}{\Lambda^{2}} \frac{\left(\partial_{\mu} \bar{z} \partial^{\mu} \bar{z}\right)(z \square z)}{\left(1+|z|^{2}\right)^{3}} \\
& \left.+\frac{c_{4 \square}-2 \alpha}{\Lambda^{2}} \frac{\square z \square \bar{z}}{\left(1+|z|^{2}\right)^{2}}+\mathcal{O}\left(\Lambda^{-4}\right)\right\} .
\end{aligned}
$$

Thus setting $\alpha=\frac{1}{2} c_{4 \square}$ and $\gamma=-c_{4 \square}$, we get

$$
\begin{aligned}
\mathcal{L}=-2 \Lambda^{d-1}\{ & \frac{\partial_{\mu} z \partial^{\mu} \bar{z}}{\left(1+|z|^{2}\right)^{2}}+\frac{2 c_{4}+4 c_{4}^{\prime}+4 c_{4} \square}{\Lambda^{2}} \frac{\left(\partial_{\mu} z \partial^{\mu} \bar{z}\right)\left(\partial_{\nu} z \partial^{\nu} \bar{z}\right)}{\left(1+|z|^{2}\right)^{4}} \\
& \left.-\frac{2 c_{4}}{\Lambda^{2}} \frac{\left(\partial_{\mu} z \partial^{\mu} z\right)\left(\partial_{\nu} \bar{z} \partial^{\nu} \bar{z}\right)}{\left(1+|z|^{2}\right)^{4}}\right\},
\end{aligned}
$$

which is thus a box-free Lagrangian density. It is now easy to see, by comparing the coefficients of the above equation with those of eq. (3.23), that transforming back to vector coordinates $\mathbf{n}$, yields the theory (3.22). 
Remark 7 Since we have eliminated all boxes from the Lagrangian to this order, the equation of motion is of second order - both in time and space directions. The theory to this order in $1 / \Lambda$ is thus free from the Ostrogradsky ghost or related instabilities [8], see appendix $A$.

Remark 8 The constraints $c_{4}+c_{4}^{\prime} \geq 0, c_{4}^{\prime} \geq 0$ and $c_{4 \square} \geq 0$ are due to constraining the static energy density to be positive definite, which is easier to do by considering the eigenvalues of the strain tensor $\partial_{\mu} \mathbf{n} \cdot \partial_{\nu} \mathbf{n}$. It is thus easy to see that the $-c_{4}$ term will give a positive energy as long as $c_{4}<c_{4}+c_{4}^{\prime}$ [14], hence yielding the second constraint.

\subsection{Order $\Lambda^{-4}$}

We will now consider the next order in the derivative or $1 / \Lambda$ expansion of the EFT, and thus write down the list of all possible operators to order $\Lambda^{-4}$ in the EFT expansion, which is: group 1:

$$
\begin{aligned}
& F_{11} \equiv\left(\partial_{\mu} \mathbf{n} \cdot \partial^{\mu} \mathbf{n}\right)\left(\partial_{\nu} \mathbf{n} \cdot \partial^{\nu} \mathbf{n}\right)\left(\partial_{\rho} \mathbf{n} \cdot \partial^{\rho} \mathbf{n}\right), \\
& F_{12} \equiv\left(\partial_{\mu} \mathbf{n} \cdot \partial^{\nu} \mathbf{n}\right)\left(\partial_{\nu} \mathbf{n} \cdot \partial^{\mu} \mathbf{n}\right)\left(\partial_{\rho} \mathbf{n} \cdot \partial^{\rho} \mathbf{n}\right), \\
& F_{13} \equiv\left(\partial_{\mu} \mathbf{n} \cdot \partial^{\nu} \mathbf{n}\right)\left(\partial_{\nu} \mathbf{n} \cdot \partial^{\rho} \mathbf{n}\right)\left(\partial_{\rho} \mathbf{n} \cdot \partial^{\mu} \mathbf{n}\right),
\end{aligned}
$$

group 2:

$$
\begin{aligned}
& F_{21} \equiv\left(\partial_{\mu} \mathbf{n} \cdot \partial^{\mu} \mathbf{n}\right)\left(\mathbf{n} \cdot \square^{2} \mathbf{n}\right), \\
& F_{22} \equiv\left(\partial_{\mu} \mathbf{n} \cdot \partial^{\mu} \mathbf{n}\right)\left(\partial_{\nu} \mathbf{n} \cdot \partial^{\nu} \square \mathbf{n}\right), \\
& F_{23} \equiv\left(\partial_{\mu} \mathbf{n} \cdot \partial^{\mu} \mathbf{n}\right)(\square \mathbf{n} \cdot \square \mathbf{n}), \\
& F_{24} \equiv\left(\partial_{\mu} \mathbf{n} \cdot \partial^{\mu} \mathbf{n}\right)\left(\partial_{\nu} \partial_{\rho} \mathbf{n} \cdot \partial^{\nu} \partial^{\rho} \mathbf{n}\right),
\end{aligned}
$$

group 3:

$$
\begin{aligned}
& F_{31} \equiv\left(\partial_{\mu} \mathbf{n} \cdot \partial_{\nu} \mathbf{n}\right)\left(\mathbf{n} \cdot \partial^{\mu} \partial^{\nu} \square \mathbf{n}\right), \\
& F_{32} \equiv\left(\partial_{\mu} \mathbf{n} \cdot \partial_{\nu} \mathbf{n}\right)\left(\partial^{\mu} \mathbf{n} \cdot \partial^{\nu} \square \mathbf{n}\right), \\
& F_{33} \equiv\left(\partial_{\mu} \mathbf{n} \cdot \partial_{\nu} \mathbf{n}\right)\left(\partial_{\rho} \mathbf{n} \cdot \partial^{\mu} \partial^{\nu} \partial^{\rho} \mathbf{n}\right), \\
& F_{34} \equiv\left(\partial_{\mu} \mathbf{n} \cdot \partial_{\nu} \mathbf{n}\right)\left(\partial^{\mu} \partial^{\nu} \mathbf{n} \cdot \square \mathbf{n}\right), \\
& F_{35} \equiv\left(\partial_{\mu} \mathbf{n} \cdot \partial_{\nu} \mathbf{n}\right)\left(\partial^{\mu} \partial^{\rho} \mathbf{n} \cdot \partial^{\nu} \partial^{\rho} \mathbf{n}\right),
\end{aligned}
$$

group 4:

$$
\begin{aligned}
& F_{41} \equiv\left(\partial_{\mu} \mathbf{n} \cdot \square \mathbf{n}\right)\left(\mathbf{n} \cdot \partial^{\mu} \square \mathbf{n}\right), \\
& F_{42} \equiv\left(\partial_{\mu} \mathbf{n} \cdot \square \mathbf{n}\right)\left(\partial^{\mu} \mathbf{n} \cdot \square \mathbf{n}\right), \\
& F_{43} \equiv\left(\partial_{\mu} \mathbf{n} \cdot \square \mathbf{n}\right)\left(\partial^{\nu} \mathbf{n} \cdot \partial^{\mu} \partial^{\nu} \mathbf{n}\right), \\
& F_{44} \equiv\left(\mathbf{n} \cdot \partial_{\mu} \square \mathbf{n}\right)\left(\partial_{\nu} \mathbf{n} \cdot \partial^{\mu} \partial^{\nu} \mathbf{n}\right), \\
& F_{45} \equiv\left(\partial_{\mu} \mathbf{n} \cdot \partial^{\mu} \partial^{\nu} \mathbf{n}\right)\left(\partial^{\rho} \mathbf{n} \cdot \partial_{\nu} \partial_{\rho} \mathbf{n}\right), \\
& F_{46} \equiv\left(\mathbf{n} \cdot \partial_{\mu} \square \mathbf{n}\right)\left(\mathbf{n} \cdot \partial^{\mu} \square \mathbf{n}\right),
\end{aligned}
$$


group 5:

$$
\begin{aligned}
& F_{51} \equiv\left(\mathbf{n} \cdot \partial_{\mu} \partial_{\nu} \partial_{\rho} \mathbf{n}\right)\left(\mathbf{n} \cdot \partial^{\mu} \partial^{\nu} \partial^{\rho} \mathbf{n}\right), \\
& F_{52} \equiv\left(\mathbf{n} \cdot \partial_{\mu} \partial_{\nu} \partial_{\rho} \mathbf{n}\right)\left(\partial^{\mu} \mathbf{n} \cdot \partial^{\nu} \partial^{\rho} \mathbf{n}\right), \\
& F_{53} \equiv\left(\partial_{\mu} \mathbf{n} \cdot \partial_{\nu} \partial_{\rho} \mathbf{n}\right)\left(\partial^{\mu} \mathbf{n} \cdot \partial^{\nu} \partial^{\rho} \mathbf{n}\right), \\
& F_{54} \equiv\left(\partial_{\mu} \mathbf{n} \cdot \partial_{\nu} \partial_{\rho} \mathbf{n}\right)\left(\partial^{\nu} \mathbf{n} \cdot \partial^{\mu} \partial^{\rho} \mathbf{n}\right),
\end{aligned}
$$

group 6:

$$
\begin{aligned}
& F_{61} \equiv \mathbf{n} \cdot \square^{3} \mathbf{n}, \\
& F_{62} \equiv \partial_{\mu} \mathbf{n} \cdot \partial^{\mu} \square^{2} \mathbf{n}, \\
& F_{63} \equiv \partial_{\mu} \partial_{\nu} \mathbf{n} \cdot \partial^{\mu} \partial^{\nu} \square \mathbf{n}, \\
& F_{64} \equiv \square \mathbf{n} \cdot \square^{2} \mathbf{n}, \\
& F_{65} \equiv \partial_{\mu} \partial_{\nu} \partial_{\rho} \mathbf{n} \cdot \partial^{\mu} \partial^{\nu} \partial^{\rho} \mathbf{n}, \\
& F_{66} \equiv \partial_{\mu} \square \mathbf{n} \cdot \partial^{\mu} \square \mathbf{n},
\end{aligned}
$$

group 7:

$$
\begin{aligned}
& F_{71} \equiv\left(\epsilon^{\mu \nu} \mathbf{n} \cdot \partial_{\mu} \mathbf{n} \times \partial_{\nu} \mathbf{n}\right)\left(\partial_{\mu} \mathbf{n} \cdot \partial^{\mu} \mathbf{n}\right)\left(\partial_{\nu} \mathbf{n} \cdot \partial^{\nu} \mathbf{n}\right), \\
& F_{72} \equiv\left(\epsilon^{\mu \nu} \mathbf{n} \cdot \partial_{\mu} \mathbf{n} \times \partial_{\nu} \mathbf{n}\right)\left(\partial_{\mu} \mathbf{n} \cdot \partial_{\nu} \mathbf{n}\right)\left(\partial^{\mu} \mathbf{n} \cdot \partial^{\nu} \mathbf{n}\right), \\
& F_{73} \equiv\left(\epsilon^{\mu \nu} \mathbf{n} \cdot \partial_{\mu} \mathbf{n} \times \partial_{\nu} \mathbf{n}\right)\left(\mathbf{n} \cdot \square^{2} \mathbf{n}\right), \\
& F_{74} \equiv\left(\epsilon^{\mu \nu} \mathbf{n} \cdot \partial_{\mu} \mathbf{n} \times \partial_{\nu} \mathbf{n}\right)\left(\partial_{\mu} \mathbf{n} \cdot \partial^{\mu} \square \mathbf{n}\right), \\
& F_{75} \equiv\left(\epsilon^{\mu \nu} \mathbf{n} \cdot \partial_{\mu} \mathbf{n} \times \partial_{\nu} \mathbf{n}\right)(\square \mathbf{n} \cdot \square \mathbf{n}), \\
& F_{76} \equiv\left(\epsilon^{\mu \nu} \mathbf{n} \cdot \partial_{\mu} \mathbf{n} \times \partial_{\nu} \mathbf{n}\right)\left(\partial_{\mu} \partial_{\nu} \mathbf{n} \cdot \partial^{\mu} \partial^{\nu} \mathbf{n}\right),
\end{aligned}
$$

which is a total of 34 operators and we have already eliminated operators that are related by eq. (2.27) to the ones in the above list. We have furthermore eliminated all operators that are related by the relation (3.18) to the above ones.

Performing integrations by parts on operators with four fields

$$
\begin{aligned}
\partial_{\mu}\left[\left(\partial_{\nu} \mathbf{n} \cdot \partial^{\nu} \mathbf{n}\right)\left(\mathbf{n} \cdot \partial^{\mu} \square \mathbf{n}\right)\right] & =F_{21}+F_{22}+2 F_{44}, \\
\partial_{\mu}\left[\left(\partial_{\nu} \mathbf{n} \cdot \partial^{\nu} \mathbf{n}\right)\left(\partial^{\mu} \mathbf{n} \cdot \square \mathbf{n}\right)\right] & =F_{22}+F_{23}+2 F_{43}, \\
\partial_{\mu}\left[\left(\partial_{\nu} \mathbf{n} \cdot \partial^{\nu} \mathbf{n}\right)\left(\partial_{\rho} \mathbf{n} \cdot \partial^{\mu} \partial^{\rho} \mathbf{n}\right)\right] & =F_{22}+F_{24}+2 F_{45}, \\
\partial_{\mu}\left[\left(\partial^{\mu} \mathbf{n} \cdot \partial^{\nu} \mathbf{n}\right)\left(\mathbf{n} \cdot \partial_{\nu} \square \mathbf{n}\right)\right] & =F_{31}+F_{32}+F_{41}+F_{44}, \\
\partial_{\mu}\left[\left(\partial_{\nu} \mathbf{n} \cdot \partial_{\rho} \mathbf{n}\right)\left(\mathbf{n} \cdot \partial^{\mu} \partial^{\nu} \partial^{\rho} \mathbf{n}\right)\right] & =F_{31}+F_{33}+2 F_{52}, \\
\partial_{\mu}\left[\left(\partial^{\mu} \mathbf{n} \cdot \partial^{\nu} \mathbf{n}\right)\left(\partial_{\nu} \mathbf{n} \cdot \square \mathbf{n}\right)\right] & =F_{32}+F_{34}+F_{42}+F_{43}, \\
\partial_{\mu}\left[\left(\partial_{\nu} \mathbf{n} \cdot \partial_{\rho} \mathbf{n}\right)\left(\partial^{\nu} \mathbf{n} \cdot \partial^{\mu} \partial^{\rho} \mathbf{n}\right)\right] & =F_{32}+F_{35}+F_{53}+F_{54}, \\
\partial_{\mu}\left[\left(\partial_{\nu} \mathbf{n} \cdot \partial_{\rho} \mathbf{n}\right)\left(\partial^{\mu} \mathbf{n} \cdot \partial^{\nu} \partial^{\rho} \mathbf{n}\right)\right] & =F_{33}+F_{34}+2 F_{54}, \\
\partial_{\mu}\left[\left(\partial^{\mu} \mathbf{n} \cdot \partial^{\nu} \mathbf{n}\right)\left(\partial^{\rho} \mathbf{n} \cdot \partial_{\nu} \partial_{\rho} \mathbf{n}\right)\right] & =F_{33}+F_{35}+F_{43}+F_{45}, \\
\partial_{\mu}\left[(\mathbf{n} \cdot \square \mathbf{n})\left(\mathbf{n} \cdot \partial^{\mu} \square \mathbf{n}\right)\right] & =-F_{21}-F_{22}+F_{41}+F_{46}, \\
\partial_{\mu}\left[(\mathbf{n} \cdot \square \mathbf{n})\left(\partial^{\mu} \mathbf{n} \cdot \square \mathbf{n}\right)\right] & =-F_{22}-F_{23}+F_{41}+F_{42},
\end{aligned}
$$




$$
\begin{aligned}
\partial_{\mu}\left[(\mathbf{n} \cdot \square \mathbf{n})\left(\partial_{\nu} \mathbf{n} \cdot \partial^{\mu} \partial^{\nu} \mathbf{n}\right)\right] & =-F_{22}-F_{24}+F_{43}+F_{44}, \\
\partial_{\mu}\left[\left(\mathbf{n} \cdot \partial^{\mu} \partial^{\nu} \mathbf{n}\right)\left(\mathbf{n} \cdot \partial_{\nu} \square \mathbf{n}\right)\right] & =-F_{31}-F_{32}+F_{44}+F_{46}, \\
\partial_{\mu}\left[\left(\mathbf{n} \cdot \partial_{\nu} \partial_{\rho} \mathbf{n}\right)\left(\mathbf{n} \cdot \partial^{\mu} \partial^{\nu} \partial^{\rho} \mathbf{n}\right)\right] & =-F_{31}-F_{33}+F_{51}+F_{52}, \\
\partial_{\mu}\left[\left(\mathbf{n} \cdot \partial^{\mu} \partial^{\nu} \mathbf{n}\right)\left(\partial_{\nu} \mathbf{n} \cdot \square \mathbf{n}\right)\right] & =-F_{32}-F_{34}+F_{41}+F_{43}, \\
\partial_{\mu}\left[\left(\mathbf{n} \cdot \partial_{\nu} \partial_{\rho} \mathbf{n}\right)\left(\partial^{\nu} \mathbf{n} \cdot \partial^{\mu} \partial^{\rho} \mathbf{n}\right)\right] & =-F_{32}-F_{35}+F_{52}+F_{54}, \\
\partial_{\mu}\left[\left(\mathbf{n} \cdot \partial_{\nu} \partial_{\rho} \mathbf{n}\right)\left(\partial^{\mu} \mathbf{n} \cdot \partial^{\nu} \partial^{\rho} \mathbf{n}\right)\right] & =-F_{33}-F_{34}+F_{52}+F_{53}, \\
\partial_{\mu}\left[\left(\mathbf{n} \cdot \partial^{\mu} \partial^{\nu} \mathbf{n}\right)\left(\partial^{\rho} \mathbf{n} \cdot \partial_{\nu} \partial_{\rho} \mathbf{n}\right)\right] & =-F_{33}-F_{35}+F_{44}+F_{45},
\end{aligned}
$$

we find 18 total derivatives and hence 18 relations between the dimension- 6 operators with four fields. There are 19 of such operators (i.e. eq. (3.32)-(3.50)) and 18 relations, so one would naively think that all but one can be eliminated. However, some of the relations are dependent on others; in fact the rank of the vectors in the space of coefficients is only 13, so we can only eliminate 13 operators, leaving us with 6 independent operators composed by six derivatives and four fields. There is quite a lot of ambiguity in which operators to keep and which to eliminate. Our choice of which to keep is predicated on symmetry in the derivatives and the preference of having a box instead of two uncontracted derivatives acting on the field, because of the field definitions that we have in mind, to be applied shortly.

Performing instead integrations by parts on the operators with two fields and six derivatives, we have

$$
\begin{aligned}
\partial_{\mu}\left[\mathbf{n} \cdot \partial^{\mu} \square^{2} \mathbf{n}\right] & =F_{61}+F_{62}, \\
\partial_{\mu}\left[\partial_{\nu} \mathbf{n} \cdot \partial^{\mu} \partial^{\nu} \square \mathbf{n}\right] & =F_{62}+F_{63}, \\
\partial_{\mu}\left[\partial^{\mu} \mathbf{n} \cdot \square^{2} \mathbf{n}\right] & =F_{62}+F_{64}, \\
\partial_{\mu}\left[\partial_{\nu} \partial_{\rho} \mathbf{n} \cdot \partial^{\mu} \partial^{\nu} \partial^{\rho} \mathbf{n}\right] & =F_{63}+F_{65}, \\
\partial_{\mu}\left[\square \mathbf{n} \cdot \partial^{\mu} \square \mathbf{n}\right] & =F_{64}+F_{66},
\end{aligned}
$$

and hence all but one operator can be eliminated. We will choose to retain $F_{66}$.

Lemma 9 The remaining operators after the integration by parts procedure, are a complete basis of the most general $\mathrm{O}(3)$, parity and Lorentz invariant dimension- 6 operators, and they are given by:

$$
F_{11}, F_{12}, F_{13}, F_{23}, F_{24}, F_{34}, F_{42}, F_{45}, F_{53}, F_{66},
$$

of eqs. (3.34), (3.35), (3.39), (3.42), (3.45), (3.49) and (3.56), respectively.

Proof. First we will write the Lagrangian as

$$
\begin{aligned}
\mathcal{L}^{(4)}= & c_{11} F_{11}+c_{12} F_{12}+c_{13} F_{13} \\
& +c_{21} F_{21}+c_{22} F_{22}+c_{23} F_{23}+c_{24} F_{24} \\
& +c_{31} F_{31}+c_{32} F_{32}+c_{33} F_{33}+c_{34} F_{34}+c_{35} F_{35} \\
& +c_{41} F_{41}+c_{42} F_{42}+c_{43} F_{43}+c_{44} F_{44}+c_{45} F_{45}+c_{46} F_{46} \\
& +c_{51} F_{51}+c_{52} F_{52}+c_{53} F_{53}+c_{54} F_{54} \\
& +c_{61} F_{61}+c_{62} F_{62}+c_{63} F_{63}+c_{64} F_{64}+c_{65} F_{65}+c_{66} F_{66} .
\end{aligned}
$$


Using eqs. (3.63) we obtain

$$
\begin{aligned}
& F_{21}=-F_{23}+2 F_{24}+8 F_{45}, \\
& F_{22}=-F_{24}-2 F_{45}, \\
& F_{31}=-2 F_{23}+2 F_{24}+F_{34}+2 F_{42}+6 F_{45}, \\
& F_{32}=\frac{F_{23}}{2}-\frac{F_{24}}{2}-F_{34}-F_{42}-F_{45}, \\
& F_{33}=\frac{2}{3} F_{23}-\frac{2}{3} F_{24}-F_{34}-\frac{2}{3} F_{42}-2 F_{45}+\frac{2}{3} F_{53}, \\
& F_{35}=-\frac{F_{23}}{6}+\frac{F_{24}}{6}+F_{34}+\frac{2}{3} F_{42}-\frac{2}{3} F_{53}, \\
& F_{41}=F_{23}-F_{24}-F_{42}-2 F_{45}, \\
& F_{43}=-\frac{F_{23}}{2}+\frac{F_{24}}{2}+F_{45}, \\
& F_{44}=\frac{F_{23}}{2}-\frac{F_{24}}{2}-3 F_{45}, \\
& F_{46}=-2 F_{23}+2 F_{24}+F_{42}+8 F_{45}, \\
& F_{51}=-2 F_{23}+2 F_{24}+2 F_{42}+6 F_{45}+F_{53}, \\
& F_{52}=\frac{2}{3} F_{23}-\frac{2}{3} F_{24}-\frac{2}{3} F_{42}-2 F_{45}-\frac{F_{53}}{6}, \\
& F_{54}=-\frac{F_{23}}{3}+\frac{F_{24}}{3}+\frac{F_{42}}{3}+F_{45}-\frac{F_{53}}{3},
\end{aligned}
$$

up to total derivatives and using eqs. (3.64), we get

$$
F_{61}=-F_{66}, \quad F_{62}=F_{66}, \quad F_{63}=-F_{66}, \quad F_{64}=-F_{66}, \quad F_{65}=F_{66},
$$

up to total derivatives. Hence, we can write

$$
\begin{aligned}
\mathcal{L}^{(4)}= & c_{11} F_{11}+c_{12} F_{12}+c_{13} F_{13} \\
& +c_{23}^{\prime} F_{23}+c_{24}^{\prime} F_{24}+c_{34}^{\prime} F_{34}+c_{42}^{\prime} F_{42}+c_{45}^{\prime} F_{45}+c_{53}^{\prime} F_{53}+c_{66}^{\prime} F_{66},
\end{aligned}
$$

with the new coefficients

$$
\begin{aligned}
c_{23}^{\prime}= & c_{23}-c_{21}-2 c_{31}+\frac{c_{32}}{2}+\frac{2}{3} c_{33}-\frac{c_{35}}{6}+c_{41}-\frac{c_{43}}{2}+\frac{c_{44}}{2}-2 c_{46}-2 c_{51}+\frac{2}{3} c_{52}-\frac{c_{54}}{3}, \\
c_{24}^{\prime}= & c_{24}+2 c_{21}-c_{22}+2 c_{31}-\frac{c_{32}}{2}-\frac{2}{3} c_{33}+\frac{c_{35}}{6}-c_{41}+\frac{c_{43}}{2}-\frac{c_{44}}{2}+2 c_{46}+2 c_{51}-\frac{2}{3} c_{52} \\
& +\frac{c_{54}}{3}, \\
c_{34}^{\prime}= & c_{34}+c_{31}-c_{32}-c_{33}+c_{35}, \\
c_{42}^{\prime}= & c_{42}+2 c_{31}-c_{32}-\frac{2}{3} c_{33}+\frac{2}{3} c_{35}-c_{41}+c_{46}+2 c_{51}-\frac{2}{3} c_{52}+\frac{c_{54}}{3}, \\
c_{45}^{\prime}= & c_{45}+8 c_{21}-2 c_{22}+6 c_{31}-c_{32}-2 c_{33}-2 c_{41}+c_{43}-3 c_{44}+8 c_{46}+6 c_{51}-2 c_{52}+c_{54}, \\
c_{53}^{\prime}= & c_{53}+\frac{2}{3} c_{33}-\frac{2}{3} c_{35}+c_{51}-\frac{c_{52}}{3}-\frac{c_{54}}{3}, \\
c_{66}^{\prime}= & c_{66}-c_{61}+c_{62}-c_{63}-c_{64}+c_{65} .
\end{aligned}
$$


The remaining operators $(F \mathrm{~s})$ in the Lagrangian (3.69) are indeed those of eq. (3.65).

At this point, it will prove convenient to introduce the following short-hand notation:

$$
\begin{aligned}
M & \equiv 1+|z|^{2} \\
K & \equiv \partial_{\mu} z \partial^{\mu} \bar{z} \\
H & \equiv \partial_{\mu} z \partial^{\mu} z \\
B & \equiv \square z \\
E & \equiv\left(\partial_{\mu} \partial_{\nu} z\right)\left(\partial^{\mu} \partial^{\nu} z\right) \\
F & \equiv\left(\partial_{\mu} \partial_{\nu} z\right)\left(\partial^{\mu} \partial^{\nu} \bar{z}\right) \\
G & \equiv\left(\partial_{\mu} \bar{z}\right)\left(\partial_{\nu} \bar{z}\right)\left(\partial^{\mu} \partial^{\nu} z\right) \\
I & \equiv\left(\partial_{\mu} z\right)\left(\partial_{\nu} \bar{z}\right)\left(\partial^{\mu} \partial^{\nu} z\right) \\
J & \equiv\left(\partial_{\mu} z\right)\left(\partial_{\nu} z\right)\left(\partial^{\mu} \partial^{\nu} z\right) \\
O & \equiv\left(\partial_{\mu} \partial_{\nu} z\right)\left(\partial^{\nu} \partial^{\rho} \bar{z}\right)\left(\partial_{\rho} \bar{z}\right)\left(\partial^{\mu} z\right), \\
P & \equiv\left(\partial_{\mu} \partial_{\nu} z\right)\left(\partial^{\nu} \partial^{\rho} \bar{z}\right)\left(\partial_{\rho} z\right)\left(\partial^{\mu} \bar{z}\right), \\
Q & \equiv\left(\partial_{\mu} \partial_{\nu} z\right)\left(\partial^{\nu} \partial^{\rho} z\right)\left(\partial_{\rho} \bar{z}\right)\left(\partial^{\mu} \bar{z}\right), \\
R & \equiv \partial_{\nu} B \partial^{\nu} \bar{z}=\left(\partial^{\nu} \square z\right)\left(\partial_{\nu} \bar{z}\right), \\
S & \equiv \partial_{\nu} B \partial^{\nu} z=\left(\partial^{\nu} \square z\right)\left(\partial_{\nu} z\right), \\
T & \equiv \frac{1}{2} \partial_{\nu} B \partial^{\nu} \bar{H}=\left(\partial^{\nu} \square z\right)\left(\partial_{\nu} \partial_{\rho} \bar{z}\right)\left(\partial^{\rho} \bar{z}\right), \\
U & \equiv \partial_{\nu} B \partial^{\nu} \bar{B}=\left(\partial^{\nu} \square z\right)\left(\partial_{\nu} \square \bar{z}\right), \\
V & \equiv\left(\partial_{\mu} z\right)\left(\partial_{\nu} z\right)\left(\partial_{\rho} \bar{z}\right)\left(\partial^{\mu} \partial^{\nu} \partial^{\rho} \bar{z}\right),
\end{aligned}
$$

where $M, K, F, O, P$ and $U$ are real Lorentz scalar quantities, whereas $H, B, E, G, I, J$, $Q, R, S, T$, and $V$ are complex Lorentz scalars. According to Theorem 6 , the order $\Lambda^{-2}$ Lagrangian in the above short-hand notation thus neatly reads

$$
\mathcal{L}=-2 \Lambda^{d-1}\left\{\frac{K}{M^{2}}+\frac{2 c_{4}+4 c_{4}^{\prime}+4 c_{4} \square}{\Lambda^{2}} \frac{K^{2}}{M^{4}}-\frac{2 c_{4}}{\Lambda^{2}} \frac{H \bar{H}}{M^{4}}+\mathcal{O}\left(\Lambda^{-4}\right)\right\} .
$$

The $\mathrm{NL} \sigma \mathrm{M}$ with $\mathrm{O}(3)$, parity and Lorentz invariance having a single mass scale $\Lambda$ to order $\Lambda^{-4}$, written as

$$
\begin{aligned}
\mathcal{L}=-\frac{\Lambda^{d-1}}{2}\{ & \left(\partial_{\mu} \mathbf{n} \cdot \partial^{\mu} \mathbf{n}\right)+\frac{c_{4}+c_{4}^{\prime}}{\Lambda^{2}}\left(\partial_{\mu} \mathbf{n} \cdot \partial^{\mu} \mathbf{n}\right)\left(\partial_{\nu} \mathbf{n} \cdot \partial^{\nu} \mathbf{n}\right)-\frac{c_{4}}{\Lambda^{2}}\left(\partial_{\mu} \mathbf{n} \cdot \partial_{\nu} \mathbf{n}\right)\left(\partial^{\mu} \mathbf{n} \cdot \partial^{\nu} \mathbf{n}\right) \\
& +\frac{c_{4} \square}{\Lambda^{2}}(\square \mathbf{n} \cdot \square \mathbf{n})+\frac{c_{11}}{\Lambda^{4}}\left(\partial_{\mu} \mathbf{n} \cdot \partial^{\mu} \mathbf{n}\right)\left(\partial_{\nu} \mathbf{n} \cdot \partial^{\nu} \mathbf{n}\right)\left(\partial_{\rho} \mathbf{n} \cdot \partial^{\rho} \mathbf{n}\right) \\
& +\frac{c_{12}}{\Lambda^{4}}\left(\partial_{\mu} \mathbf{n} \cdot \partial^{\nu} \mathbf{n}\right)\left(\partial_{\nu} \mathbf{n} \cdot \partial^{\mu} \mathbf{n}\right)\left(\partial_{\rho} \mathbf{n} \cdot \partial^{\rho} \mathbf{n}\right) \\
& +\frac{c_{13}}{\Lambda^{4}}\left(\partial_{\mu} \mathbf{n} \cdot \partial^{\nu} \mathbf{n}\right)\left(\partial_{\nu} \mathbf{n} \cdot \partial^{\rho} \mathbf{n}\right)\left(\partial_{\rho} \mathbf{n} \cdot \partial^{\mu} \mathbf{n}\right)+\frac{c_{23}}{\Lambda^{4}}\left(\partial_{\mu} \mathbf{n} \cdot \partial^{\mu} \mathbf{n}\right)(\square \mathbf{n} \cdot \square \mathbf{n}) \\
& +\frac{c_{24}}{\Lambda^{4}}\left(\partial_{\mu} \mathbf{n} \cdot \partial^{\mu} \mathbf{n}\right)\left(\partial_{\nu} \partial_{\rho} \mathbf{n} \cdot \partial^{\nu} \partial^{\rho} \mathbf{n}\right)+\frac{c_{34}}{\Lambda^{4}}\left(\partial_{\mu} \mathbf{n} \cdot \partial_{\nu} \mathbf{n}\right)\left(\partial^{\mu} \partial^{\nu} \mathbf{n} \cdot \square \mathbf{n}\right)
\end{aligned}
$$




$$
\begin{aligned}
& +\frac{c_{42}}{\Lambda^{4}}\left(\partial_{\mu} \mathbf{n} \cdot \square \mathbf{n}\right)\left(\partial^{\mu} \mathbf{n} \cdot \square \mathbf{n}\right)+\frac{c_{45}}{\Lambda^{4}}\left(\partial_{\mu} \mathbf{n} \cdot \partial^{\mu} \partial^{\nu} \mathbf{n}\right)\left(\partial^{\rho} \mathbf{n} \cdot \partial_{\nu} \partial_{\rho} \mathbf{n}\right) \\
& +\frac{c_{53}}{\Lambda^{4}}\left(\partial_{\mu} \mathbf{n} \cdot \partial_{\nu} \partial_{\rho} \mathbf{n}\right)\left(\partial^{\mu} \mathbf{n} \cdot \partial^{\nu} \partial^{\rho} \mathbf{n}\right)+\frac{c_{66}}{\Lambda^{4}}\left(\partial_{\mu} \square \mathbf{n} \cdot \partial^{\mu} \square \mathbf{n}\right)+\mathcal{O}\left(\Lambda^{-6}\right) \\
& +\lambda(\mathbf{n} \cdot \mathbf{n}-1)\}
\end{aligned}
$$

subject to the constraints $c_{4}+c_{4}^{\prime} \geq 0, c_{4}^{\prime} \geq 0, c_{4 \square} \geq 0, c_{11} \geq 0, c_{12} \geq 0, c_{13} \geq 0, c_{23} \geq 0$, $c_{24} \geq 0, c_{34} \geq 0, c_{42} \geq 0, c_{45} \geq 0, c_{53} \geq 0, c_{66} \geq 0$ can be written in terms of the Riemann sphere coordinate $z$ and its complex conjugate as

$$
\mathcal{L}=-2 \Lambda^{d-1}\left\{\mathcal{L}^{(0)}+\frac{1}{\Lambda^{2}} \mathcal{L}^{(2)}+\frac{1}{\Lambda^{4}} \mathcal{L}^{(4)}+\mathcal{O}\left(\Lambda^{-6}\right)\right\}
$$

with the respective orders

$$
\begin{aligned}
& \mathcal{L}^{(0)}=\frac{K}{M^{2}}, \\
& \mathcal{L}^{(2)}=\left(2 c_{4}+4 c_{4}^{\prime}+4 c_{4 \square}\right) \frac{K^{2}}{M^{4}}-\left(2 c_{4}+4 c_{4 \square}\right) \frac{H \bar{H}}{M^{4}}-2 c_{4 \square} \frac{H \bar{z} \bar{B}}{M^{3}}-2 c_{4 \square} \frac{\bar{H} z B}{M^{3}}+4 c_{4 \square} \frac{H \bar{H}}{M^{3}} \\
& +c_{4 \square} \frac{B \bar{B}}{M^{2}}, \\
& \mathcal{L}^{(4)}=c_{\{6,5\}}^{K^{3}}\left[\frac{K^{3}}{M^{\{6,5\}}}\right]+c_{\{6,5,4\}}^{K H \bar{H}}\left[\frac{K H \bar{H}}{M^{\{6,5,4\}}}\right]+c_{6}^{z^{2} H \bar{H}^{2}}\left[\frac{z^{2} H \bar{H}^{2}+\bar{z}^{2} H^{2} \bar{H}}{M^{6}}\right] \\
& +c_{6}^{\bar{z}^{2} K^{2} H}\left[\frac{\bar{z}^{2} K^{2} H+z^{2} K^{2} \bar{H}}{M^{6}}\right]+c_{\{5,4\}}^{\bar{z} K H \bar{B}}\left[\frac{\bar{z} K H \bar{B}+z K \bar{H} B}{M^{\{5,4\}}}\right]+c_{\{4,3\}}^{K B \bar{B}}\left[\frac{K B \bar{B}}{M^{\{4,3\}}}\right] \\
& -4 c_{42}\left[\frac{\bar{z} H \bar{H} B+z H \bar{H} \bar{B}}{M^{5}}\right]+c_{4}^{H \bar{B}^{2}}\left[\frac{H \bar{B}^{2}+\bar{H} B^{2}}{M^{4}}\right]+c_{53}\left[\frac{H \bar{E}+\bar{H} E}{M^{4}}\right] \\
& +c_{4}^{K F}\left[\frac{K F}{M^{4}}\right]+c_{5}^{z K G}\left[\frac{z K G+\bar{z} K \bar{G}}{M^{5}}\right]-8 c_{66}\left[\frac{\bar{z} H G+z \bar{H} \bar{G}}{M^{5}}\right]+4 c_{66}\left[\frac{B G+\bar{B} \bar{G}}{M^{4}}\right] \\
& -4 c_{45}\left[\frac{\bar{z} K I+z K \bar{I}}{M^{5}}\right]+c_{\{5,4\}}^{z \bar{H} I}\left[\frac{z \bar{H} I+\bar{z} H \bar{I}}{M^{\{5,4\}}}\right]+c_{\{4,3\}}^{\bar{B} I}\left[\frac{\bar{B} I+B \bar{I}}{M^{\{4,3\}}}\right] \\
& +c_{5}^{z H \bar{J}}\left[\frac{z H \bar{J}+\bar{z} \bar{H} J}{M^{5}}\right]+16 c_{66}\left[\frac{|z|^{2} O}{M^{4}}\right]+c_{4}^{P}\left[\frac{P}{M^{4}}\right]+c_{4}^{Q}\left[\frac{Q+\bar{Q}}{M^{4}}\right] \\
& -4 c_{66}\left[\frac{K R+K \bar{R}}{M^{4}}\right]+6 c_{66}\left[\frac{\bar{z}^{2} H \bar{R}+z^{2} \bar{H} R}{M^{4}}\right]-2 c_{66}\left[\frac{z \bar{B} R+\bar{z} B \bar{R}}{M^{3}}\right] \\
& -2 c_{66}\left[\frac{H \bar{S}+\bar{H} S}{M^{4}}\right]-8 c_{66}\left[\frac{z T+\bar{z} \bar{T}}{M^{3}}\right]+c_{66}\left[\frac{U}{M^{2}}\right] \text {, }
\end{aligned}
$$

with coefficients

$$
\begin{aligned}
c_{6}^{K^{3}} & =16 c_{11}+8 c_{12}+4 c_{13}+16 c_{23}-8 c_{24}+8 c_{34}-8 c_{45}-8 c_{53}-16 c_{66}, \\
c_{5}^{K^{3}} & =16 c_{24}+8 c_{45}+8 c_{53}+32 c_{66}, \\
c_{6}^{K H} \bar{H} & =8 c_{12}+12 c_{13}-16 c_{23}+8 c_{24}-8 c_{34}-8 c_{42}+16 c_{66},
\end{aligned}
$$




$$
\begin{aligned}
c_{5}^{K H \bar{H}} & =16 c_{23}+16 c_{34}+8 c_{42}-32 c_{66}, \\
c_{4}^{K H \bar{H}} & =36 c_{66}, \\
c_{6}^{z^{2} H \bar{H}^{2}} & =4 c_{42}+4 c_{53}-8 c_{66}, \\
c_{6}^{\bar{z}^{2} K^{2} H} & =4 c_{45}+8 c_{66}, \\
c_{5}^{\bar{z} K H \bar{B}} & =-8 c_{23}-4 c_{34}-4 c_{42}, \\
c_{4}^{\bar{z} K H \bar{B}} & =-12 c_{66} \\
c_{4}^{K B \bar{B}} & =4 c_{23}+2 c_{42}-2 c_{66}, \\
c_{3}^{K B \bar{B}} & =4 c_{66}, \\
c_{4}^{H \bar{B}^{2}} & =c_{42}+c_{66}, \\
c_{4}^{K F} & =4 c_{24}+2 c_{53}, \\
c_{5}^{z K G} & =-8 c_{24}-4 c_{45}-4 c_{53}-16 c_{66}, \\
c_{5}^{z \bar{H} I} & =-4 c_{34}+16 c_{66}, \\
c_{4}^{z \bar{H} I} & =-24 c_{66}, \\
c_{4}^{\bar{B} I} & =2 c_{34}-4 c_{66}, \\
c_{3}^{\bar{B} I} & =8 c_{66}, \\
c_{5}^{z H \bar{J}} & =-4 c_{53}+8 c_{66}, \\
c_{4}^{P} & =2 c_{45}+8 c_{66}, \\
c_{4}^{Q} & =c_{45}+4 c_{66},
\end{aligned}
$$

where the short-hand notation (3.71) has been used and we have defined the notation

$$
c_{\{a, b, c\}} \frac{X}{M^{\{a, b, c\}}}=\sum_{n \in\{a, b, c\}} c_{n} \frac{X}{M^{n}} .
$$

We will now perform field redefinitions of the field $z$ up to order $\Lambda^{-4}$ :

$$
z \rightarrow z+\frac{1}{\Lambda^{2}} \psi_{1}+\frac{1}{\Lambda^{4}} \psi_{2},
$$

where $\psi_{1}$ was already determined in Theorem 6 and in the short-hand notation (3.71) is given by

$$
\psi_{1}=c_{4 \square}\left(\frac{1}{2} B-\frac{H \bar{z}}{M}\right),
$$

whereas $\psi_{2}$ is only determined at the higher order, namely at order $\Lambda^{-4}$. The zeroth order Lagrangian is unchanged under any field redefinition (by definition), whereas the proof of Theorem 6 can be restated in the short-hand notation (3.71) as

$$
\begin{aligned}
\delta \mathcal{L}^{(2)} & =\frac{\partial \mathcal{L}^{(0)}}{\partial z} \psi_{1}+\frac{\partial \mathcal{L}^{(0)}}{\partial \bar{z}} \bar{\psi}_{1}-\partial_{\mu}\left(\frac{\partial \mathcal{L}^{(0)}}{\partial \partial_{\mu} z}\right) \psi_{1}-\partial_{\mu}\left(\frac{\partial \mathcal{L}^{(0)}}{\partial \partial_{\mu} \bar{z}}\right) \bar{\psi}_{1} \\
& =\left(-\frac{\bar{B}}{M^{2}}+\frac{2 \bar{H} z}{M^{3}}\right) \psi_{1}+\left(-\frac{B}{M^{2}}+\frac{2 H \bar{z}}{M^{3}}\right) \bar{\psi}_{1}
\end{aligned}
$$


and hence we obtain nicely

$$
\mathcal{L}^{(2)}+\delta \mathcal{L}^{(2)}=\left(2 c_{4}+4 c_{4}^{\prime}+4 c_{4 \square}\right) \frac{K^{2}}{M^{4}}-2 c_{4} \frac{H \bar{H}}{M^{4}},
$$

which indeed is box free.

The change of the fourth-order Lagrangian due to the field redefinition (3.80) is given by

$$
\begin{aligned}
\delta \mathcal{L}^{(4)}= & \frac{\partial \mathcal{L}^{(0)}}{\partial z} \psi_{2}+\frac{\partial \mathcal{L}^{(0)}}{\partial \bar{z}} \bar{\psi}_{2}+\frac{\partial \mathcal{L}^{(0)}}{\partial \partial_{\mu} z} \partial_{\mu} \psi_{2}+\frac{\partial \mathcal{L}^{(0)}}{\partial \partial_{\mu} \bar{z}} \partial_{\mu} \bar{\psi}_{2} \\
& +\frac{1}{2} \frac{\partial^{2} \mathcal{L}^{(0)}}{\partial z^{2}} \psi_{1}^{2}+\frac{1}{2} \frac{\partial^{2} \mathcal{L}^{(0)}}{\partial \bar{z}^{2}} \bar{\psi}_{1}^{2}+\frac{\partial^{2} \mathcal{L}^{(0)}}{\partial z \partial \bar{z}}\left|\psi_{1}\right|^{2}+\frac{\partial^{2} \mathcal{L}^{(0)}}{\partial \partial_{\mu} z \partial \partial_{\nu} \bar{z}} \partial_{\mu} \psi_{1} \partial_{\nu} \bar{\psi}_{1} \\
& +\frac{\partial^{2} \mathcal{L}^{(0)}}{\partial \partial_{\mu} z \partial z} \psi_{1} \partial_{\mu} \psi_{1}+\frac{\partial^{2} \mathcal{L}^{(0)}}{\partial \partial_{\mu} z \partial \bar{z}} \bar{\psi}_{1} \partial_{\mu} \psi_{1}+\frac{\partial^{2} \mathcal{L}^{(0)}}{\partial \partial_{\mu} \bar{z} \partial z} \psi_{1} \partial_{\mu} \bar{\psi}_{1}+\frac{\partial^{2} \mathcal{L}^{(0)}}{\partial \partial_{\mu} \bar{z} \partial \bar{z}} \bar{\psi}_{1} \partial_{\mu} \bar{\psi}_{1} \\
& +\frac{\partial \mathcal{L}^{(2)}}{\partial z} \psi_{1}+\frac{\partial \mathcal{L}^{(2)}}{\partial \bar{z}} \bar{\psi}_{1}+\frac{\partial \mathcal{L}^{(2)}}{\partial \partial_{\mu} z} \partial_{\mu} \psi_{1}+\frac{\partial \mathcal{L}^{(2)}}{\partial \partial_{\mu} \bar{z}} \partial_{\mu} \bar{\psi}_{1}+\frac{\partial \mathcal{L}^{(2)}}{\partial \square z} \square \psi_{1}+\frac{\partial \mathcal{L}^{(2)}}{\partial \square \bar{z}} \square \bar{\psi}_{1} .
\end{aligned}
$$

Remark 10 The corrections to $\mathcal{L}^{(2)}$ due to field redefinitions are determined only by $\psi_{1}$. $\psi_{1}$ in turn will affect $\mathcal{L}^{(4)}$, but since we fixed $\psi_{1}$ at the previous order, the impact at order $\Lambda^{-4}$ and hence on $\mathcal{L}^{(4)}$ is given. $\psi_{2}$ does not affect $\mathcal{L}^{(2)}$ and should thus be used for simplifying $\mathcal{L}^{(4)}$. The perturbative ordering in the field redefinitions is thus evident.

The $\mathrm{NL} \sigma \mathrm{M}$ with $\mathrm{O}(3)$, parity and Lorentz invariance having a single mass scale $\Lambda$ to order $\Lambda^{-4}$ (3.74) with (3.75)-(3.77) and with the field redefinition (3.80) and $\psi_{1}$ of eq. (3.81) is given by Lagrangian (3.74) with the zeroth order unchanged (eq. (3.75)), the second order in $1 / \Lambda$ by eq. (3.83) and the fourth order in $1 / \Lambda$ by

$$
\begin{aligned}
& \mathcal{L}^{(4)}+\delta \mathcal{L}^{(4)}=c_{\{6,5\}}^{K^{3}}\left[\frac{K^{3}}{M^{\{6,5\}}}\right]+c_{\{6,5,4\}}^{K H \bar{H}}\left[\frac{K H \bar{H}}{M^{\{6,5,4\}}}\right]+5 c_{4 \square}^{2}\left[\frac{z^{4} K \bar{H}^{2}+\bar{z}^{4} K H^{2}}{M^{6}}\right] \\
& +c_{\{6,5\}}^{z^{2} H \bar{H}^{2}}\left[\frac{z^{2} H \bar{H}^{2}+\bar{z}^{2} \bar{H} H^{2}}{M^{\{6,5\}}}\right]+c_{6}^{\bar{z}^{2} K^{2} H}\left[\frac{\bar{z}^{2} K^{2} H+z^{2} K^{2} \bar{H}}{M^{6}}\right] \\
& +c_{\{5,4\}}^{\bar{z} K H \bar{B}}\left[\frac{\bar{z} K H \bar{B}+z K \bar{H} B}{M^{\{5,4\}}}\right]-4 c_{4 \square}^{2}\left[\frac{z^{3} K \bar{H} \bar{B}+\bar{z}^{3} K H B}{M^{5}}\right]+c_{\{4,3\}}^{K B \bar{B}}\left[\frac{K B \bar{B}}{M^{\{4,3\}}}\right] \\
& +\frac{3}{4} c_{4 \square}^{2}\left[\frac{z^{2} K \bar{B}^{2}+\bar{z}^{2} K B^{2}}{M^{4}}\right]+c_{5}^{z K^{2} \bar{B}}\left[\frac{z K^{2} \bar{B}+\bar{z} K^{2} B}{M^{5}}\right]+c_{\{5,4\}}^{\bar{z} H \bar{H} B}\left[\frac{\bar{z} H \bar{H} B+z H \bar{H} \bar{B}}{M^{\{5,4\}}}\right] \\
& +c_{\{4,3\}}^{H \bar{B}^{2}}\left[\frac{H \bar{B}^{2}+\bar{H} B^{2}}{M^{\{4,3\}}}\right]-c_{4 \square}^{2}\left[\frac{z B \bar{B}^{2}+\bar{z} \bar{B} B^{2}}{M^{3}}\right]+5 c_{4 \square}^{2}\left[\frac{z^{2} \bar{H} B \bar{B}+\bar{z}^{2} H B \bar{B}}{M^{4}}\right] \\
& -8 c_{4 \square}^{2}\left[\frac{z^{3} \bar{H}^{2} B+\bar{z}^{3} H^{2} \bar{B}}{M^{5}}\right]+c_{53}\left[\frac{H \bar{E}+\bar{H} E}{M^{4}}\right]+c_{4}^{K F} \frac{K F}{M^{4}}+c_{5}^{z K G}\left[\frac{z K G+\bar{z} K \bar{G}}{M^{5}}\right] \\
& -8 c_{66}\left[\frac{\bar{z} H G+z \bar{H} \bar{G}}{M^{5}}\right]+4 c_{66}\left[\frac{B G+\bar{B} \bar{G}}{M^{4}}\right]+c_{5}^{\bar{z} K I}\left[\frac{\bar{z} K I+z K \bar{I}}{M^{5}}\right]+c_{\{5,4\}}^{z \bar{H} I}\left[\frac{z \bar{H} I+\bar{z} H \bar{I}}{M^{\{5,4\}}}\right]
\end{aligned}
$$




$$
\begin{aligned}
& +c_{\{4,3\}}^{\bar{B} I}\left[\frac{\bar{B} I+B \bar{I}}{M^{\{4,3\}}}\right]+2 c_{4 \square}^{2}\left[\frac{z^{2} \bar{B} \bar{I}+\bar{z}^{2} B I}{M^{4}}\right]-4 c_{4 \square}^{2}\left[\frac{z^{3} \bar{H} \bar{I}}{M^{5}}+\frac{\bar{z}^{3} H I}{M^{5}}\right] \\
& +c_{\{5,4\}}^{z H \bar{J}}\left[\frac{z H \bar{J}+\bar{z} \bar{H} J}{M^{\{5,4\}}}\right]+4 c_{4 \square}^{2}\left[\frac{z^{2} \bar{J} B+\bar{z}^{2} J \bar{B}}{M^{4}}\right]+c_{4}^{|z|^{2} O}\left[\frac{|z|^{2} O}{M^{4}}\right]+c_{4}^{P}\left[\frac{P}{M^{4}}\right] \\
& +c_{4}^{Q}\left[\frac{Q+\bar{Q}}{M^{4}}\right]+c_{4}^{K R}\left[\frac{K R+K \bar{R}}{M^{4}}\right]+c_{4}^{\bar{z}^{2} H \bar{R}}\left[\frac{\bar{z}^{2} H \bar{R}+z^{2} \bar{H} R}{M^{4}}\right]+c_{4 \square}^{2}\left[\frac{z^{2} \bar{H} \bar{R}+\bar{z}^{2} H R}{M^{4}}\right] \\
& +c_{3}^{z \bar{B} R}\left[\frac{z \bar{B} R+\bar{z} B \bar{R}}{M^{3}}\right]-\frac{1}{2} c_{4 \square}^{2}\left[\frac{z \bar{B} \bar{R}+\bar{z} B R}{M^{3}}\right]+c_{\{4,3\}}^{H \bar{S}}\left[\frac{H \bar{S}+\bar{H} S}{M^{\{4,3\}}}\right]-c_{4 \square}^{2}\left[\frac{z B \bar{S}+\bar{z} \bar{B} S}{M^{3}}\right] \\
& +c_{3}^{z T}\left[\frac{z T+\bar{z} \bar{T}}{M^{3}}\right]+c_{2}^{U}\left[\frac{U}{M^{2}}\right] \\
& -\left[\frac{\bar{B}}{M^{2}}-\frac{2 \bar{H} z}{M^{3}}\right] \psi_{2}^{\alpha}-\left[\frac{B}{M^{2}}-\frac{2 H \bar{z}}{M^{3}}\right] \bar{\psi}_{2}^{\alpha}-\left[\frac{2 K \bar{z}}{M^{3}}-\frac{\partial_{\mu} \bar{z}}{M^{2}} \partial^{\mu}\right] \psi_{2}^{\beta}-\left[\frac{2 K z}{M^{3}}-\frac{\partial_{\mu} z}{M^{2}} \partial^{\mu}\right] \bar{\psi}_{2}^{\beta} \\
& -\left[\frac{2 z \partial^{\mu} \bar{H}}{M^{3}}-\frac{\partial^{\mu} \bar{B}}{M^{2}}+\frac{2}{M^{3}}\left(\frac{3 \bar{H}}{M}-2 \bar{H}+\bar{z} \bar{B}\right) \partial^{\mu} z+\frac{2 z}{M^{3}}\left(\bar{B}-\frac{3 z \bar{H}}{M}\right) \partial^{\mu} \bar{z}\right] \partial_{\mu} \psi_{2}^{\eta} \\
& -\left[\frac{2 \bar{z} \partial^{\mu} H}{M^{3}}-\frac{\partial^{\mu} B}{M^{2}}+\frac{2}{M^{3}}\left(\frac{3 H}{M}-2 H+z B\right) \partial^{\mu} \bar{z}+\frac{2 \bar{z}}{M^{3}}\left(B-\frac{3 \bar{z} H}{M}\right) \partial^{\mu} z\right] \partial_{\mu} \bar{\psi}_{2}^{\eta},
\end{aligned}
$$

where we have defined the coefficients

$$
\begin{aligned}
c_{6}^{K^{3}} & =16 c_{11}+8 c_{12}+4 c_{13}+16 c_{23}-8 c_{24}+8 c_{34}-8 c_{45}-8 c_{53}-16 c_{66}, \\
c_{5}^{K^{3}} & =16 c_{24}+8 c_{45}+8 c_{53}+32 c_{66}, \\
c_{6}^{K H \bar{H}} & =8 c_{12}+12 c_{13}-16 c_{23}+8 c_{24}-8 c_{34}-8 c_{42}+16 c_{66}-12 c_{4 \square}^{2}-16 c_{4}^{\prime} c_{4 \square}, \\
c_{5}^{K H \bar{H}} & =16 c_{23}+16 c_{34}+8 c_{42}-32 c_{66}-4 c_{4 \square}^{2}, \\
c_{4}^{K H} & =36 c_{66}-3 c_{4 \square}^{2}, \\
c_{6}^{z^{2} H \bar{H}^{2}} & =4 c_{42}+4 c_{53}-8 c_{66}-17 c_{4 \square}^{2}-12 c_{4} c_{4 \square}, \\
c_{5}^{z^{2} H \bar{H}^{2}} & =16 c_{4 \square}^{2}, \\
c_{6}^{\bar{z}^{2} K^{2} H} & =4 c_{45}+8 c_{66}+24 c_{4 \square}^{2}+12 c_{4} c_{4 \square}+24 c_{4}^{\prime} c_{4 \square}, \\
c_{5}^{\bar{z} K H \bar{B}} & =-8 c_{23}-4 c_{34}-4 c_{42}+4 c_{4 \square}^{2}, \\
c_{4}^{\bar{z} K H \bar{B}} & =-12 c_{66}-c_{4 \square}^{2}, \\
c_{4}^{K B \bar{B}} & =4 c_{23}+2 c_{42}-2 c_{66}-\frac{3}{2} c_{4 \square}^{2}, \\
c_{3}^{K B \bar{B}} & =4 c_{66}+c_{4 \square}^{2}, \\
c_{5}^{z K^{2} \bar{B}} & =-8 c_{4 \square}^{2}-4 c_{4} c_{4 \square}-8 c_{4}^{\prime} c_{4 \square}, \\
c_{5}^{\bar{z} H \bar{H} B} & =-4 c_{42}+14 c_{4 \square}^{2}+4 c_{4} c_{4 \square}, \\
c_{4}^{\bar{z} H \bar{H} B} & =-10 c_{4 \square}^{2}, \\
c_{4}^{H} \bar{B}^{2} & =c_{42}+c_{66}-3 c_{4 \square}^{2}, \\
c_{3}^{H} \bar{B}^{2} & =2 c_{4 \square}^{2},
\end{aligned}
$$




$$
\begin{aligned}
c_{4}^{K F} & =4 c_{24}+2 c_{53}, \\
c_{5}^{z K G} & =-8 c_{24}-4 c_{45}-4 c_{53}-16 c_{66}, \\
c_{5}^{\bar{z} K I} & =-4 c_{45}-16 c_{4 \square}^{2}-8 c_{4} c_{4 \square}-16 c_{4}^{\prime} c_{4 \square}, \\
c_{5}^{z \bar{H} I} & =-4 c_{34}+16 c_{66}-10 c_{4 \square}^{2}, \\
c_{4}^{z \bar{H} I} & =-24 c_{66}+10 c_{4 \square}^{2}, \\
c_{4}^{\bar{B} I} & =2 c_{34}-4 c_{66}+2 c_{4 \square}^{2}, \\
c_{3}^{\bar{B} I} & =8 c_{66}-2 c_{4 \square}^{2}, \\
c_{5}^{z H \bar{J}} & =-4 c_{53}+8 c_{66}+2 c_{4 \square}^{2}+8 c_{4} c_{4 \square}, \\
c_{4}^{z H \bar{J}} & =-8 c_{4 \square}^{2}, \\
c_{4}^{|z|^{2} O} & =16 c_{66}-12 c_{4 \square}^{2}, \\
c_{4}^{P} & =2 c_{45}+8 c_{66}, \\
c_{4}^{Q} & =c_{45}+4 c_{66}, \\
c_{4}^{K R} & =-4 c_{66}+4 c_{4 \square}^{2}+2 c_{4} c_{4 \square}+4 c_{4}^{\prime} c_{4 \square}, \\
c_{4}^{\bar{z}^{2} H \bar{R}} & =6 c_{66}-\frac{5}{2} c_{4 \square}^{2}, \\
c_{3}^{z \bar{B} R} & =-2 c_{66}+\frac{1}{2} c_{4 \square}^{2}, \\
c_{4}^{H \bar{S}} & =-2 c_{66}-\frac{1}{2} c_{4 \square}^{2}-2 c_{4} c_{4 \square}, \\
c_{3}^{H} \bar{S} & =2 c_{4 \square}^{2}, \\
c_{3}^{z T} & =-8 c_{66}+6 c_{4 \square}^{2}, \\
c_{2}^{U} & =c_{66}-\frac{3}{4} c_{4 \square}^{2},
\end{aligned}
$$

and we have defined

$$
\psi_{2}=\psi_{2}^{\alpha}+\psi_{2}^{\beta}+\square \psi_{2}^{\eta},
$$

where the factor multiplying $\psi_{2}^{\alpha}$ has been integrated by parts so as to make it a multiplicative factor of $\psi_{2}^{\alpha}$, whereas the factor of $\psi_{2}^{\beta}$ has not. $\psi_{2}^{\eta}$ has been integrated by parts one more time than $\psi_{2}^{\alpha}$, which is possible as it is defined with the box. For a list of the possible terms we will use to construct $\psi_{2}^{\alpha}$ and $\psi_{2}^{\beta}$, see appendix B.

Proposition 11 The $N L \sigma M$ with $\mathrm{O}(3)$, parity and Lorentz invariance having a single mass scale $\Lambda$ to order $\Lambda^{-4}$ (3.74) with field redefinition (3.80) and Lagrangian components (3.75), (3.83) and (3.85) being zeroth, second and fourth order in $1 / \Lambda$, respectively, can be reduced to (3.74) with Lagrangian components (3.75), (3.83) and the fourth order in 
$1 / \Lambda$ given by

$$
\begin{aligned}
& \mathcal{L}^{(4)}+\delta \mathcal{L}^{(4)}=c_{\{6,5\}}^{K^{3}}\left[\frac{K^{3}}{M^{\{6,5\}}}\right]+c_{6}^{K H \bar{H}}\left[\frac{K H \bar{H}}{M^{6}}\right]+c_{6}^{z^{2} K^{2} \bar{H}}\left[\frac{z^{2} K^{2} \bar{H}+\bar{z}^{2} K^{2} H}{M^{6}}\right] \\
& -2 c_{53}\left[\frac{z^{2} H \bar{H}^{2}+\bar{z}^{2} \bar{H} H^{2}}{M^{6}}\right] \\
& +c_{53}\left[\frac{H \bar{E}+\bar{H} E}{M^{4}}\right]+c_{4}^{K F}\left[\frac{K F}{M^{4}}\right]-4 c_{53}\left[\frac{\bar{z} H G+z \bar{H} \bar{G}}{M^{5}}\right]+c_{5}^{z K \bar{I}}\left[\frac{z K \bar{I}+\bar{z} K I}{M^{5}}\right],
\end{aligned}
$$

with coefficients

$$
\begin{aligned}
c_{6}^{K^{3}} & =16 c_{11}+8 c_{12}+4 c_{13}+16 c_{23}+32 c_{24}+8 c_{34}-8 c_{45}+12 c_{53}-16 c_{66}, \\
c_{5}^{K^{3}} & =-16 c_{24}+8 c_{45}-8 c_{53}+32 c_{66}, \\
c_{6}^{K H} \bar{H} & =8 c_{12}+12 c_{13}+8 c_{24}+8 c_{34}-4 c_{45}-4 c_{53}-16 c_{66}, \\
c_{6}^{z^{2} K^{2} \bar{H}} & =-12 c_{24}+6 c_{45}+14 c_{53}+24 c_{66}, \\
c_{4}^{K F} & =4 c_{24}-2 c_{45}+2 c_{53}-8 c_{66}, \\
c_{5}^{z K \bar{I}} & =8 c_{24}-4 c_{45}-4 c_{53}-16 c_{66} .
\end{aligned}
$$

Proof. Consider the field $\psi_{2}$ with decomposition (3.87) given by

$$
\begin{aligned}
\psi_{2}^{\alpha}= & \alpha_{\{2,1\}}^{K B} \frac{K B}{M^{\{2,1\}}}+\alpha_{\{3,2\}}^{\bar{z} K H} \frac{\bar{z} K H}{M^{\{3,2\}}}+\alpha_{\{2,1\}}^{I} \frac{I}{M^{\{2,1\}}}+\alpha_{2}^{H \bar{B}} \frac{H \bar{B}}{M^{2}}+\alpha_{\{3,2\}}^{z H \bar{H}} \frac{z H \bar{H}}{M^{\{3,2\}}}+\alpha_{1}^{z R} \frac{z R}{M} \\
& -\frac{c_{4 \square}^{2}}{2} \frac{z \bar{R}}{M}+\alpha_{2}^{\bar{G}} \frac{\bar{G}}{M^{2}}+\alpha_{3}^{z K^{2}} \frac{z K^{2}}{M^{3}}+\alpha_{1}^{\bar{z} S} \frac{\bar{z} S}{M}+\alpha_{2}^{z^{2} \bar{H} B} \frac{z^{2} \bar{H} B}{M^{2}}+2 c_{4 \square}^{2} \frac{z^{2} \bar{I}}{M^{2}}+\alpha_{2}^{\bar{z}^{2} J} \frac{\bar{z}^{2} J}{M^{2}} \\
& +\frac{3}{4} c_{4 \square}^{2} \frac{z^{2} K \bar{B}}{M^{2}}+\alpha_{3}^{\bar{z}^{3} H^{2}} \frac{\bar{z}^{3} H^{2}}{M^{3}}-\frac{5}{2} c_{4 \square}^{2} \frac{z^{3} K \bar{H}}{M^{3}}+\alpha_{1}^{\bar{z} E} \frac{\bar{z} E}{M}-c_{4 \square}^{2} \frac{\bar{z} B^{2}}{M}, \\
\psi_{2}^{\beta}= & \beta_{3}^{z K^{2}} \frac{z K^{2}}{M^{3}}+\beta_{3}^{z H} \bar{H} \frac{z H \bar{H}}{M^{3}}+\beta_{3}^{\bar{z} K H} \frac{\bar{z} K H}{M^{3}}+\beta_{\{2,1\}}^{H \bar{B}} \frac{H \bar{B}}{M^{\{2,1\}}}+\beta_{1}^{z B \bar{B}} \frac{z B \bar{B}}{M}+\beta_{3}^{K B} \frac{K B}{M^{3}}, \\
\psi_{2}^{\eta}= & \left(\frac{3}{8} c_{4 \square}^{2}-\frac{1}{2} c_{66}\right) z,
\end{aligned}
$$

with coefficients

$$
\begin{aligned}
\alpha_{2}^{K B} & =2 c_{23}+c_{42}+\frac{3}{8} c_{4 \square}^{2}-\frac{5}{2} c_{66}, \\
\alpha_{1}^{K B} & =-\frac{1}{4} c_{4 \square}^{2}+3 c_{66}, \\
\alpha_{3}^{\bar{z} K H} & =-4 c_{23}-4 c_{34}-2 c_{42}-2 c_{45}-4 c_{4} c_{4 \square}+8 c_{4}^{\prime} c_{4 \square}+\frac{51}{4} c_{4 \square}^{2}-21 c_{66}, \\
\alpha_{2}^{\bar{z} K H} & =\frac{3}{4} c_{4 \square}^{2}-9 c_{66}, \\
\alpha_{2}^{I} & =2 c_{34}+c_{45}+2 c_{4} c_{4 \square}-4 c_{4}^{\prime} c_{4 \square}-\frac{13}{2} c_{4 \square}^{2}+10 c_{66}, \\
\alpha_{1}^{I} & =-\frac{1}{2} c_{4 \square}^{2}+6 c_{66},
\end{aligned}
$$




$$
\begin{aligned}
\alpha_{2}^{H \bar{B}} & =c_{42}-c_{4 \square}^{2}+c_{66}, \\
\alpha_{3}^{z H \bar{H}} & =-2 c_{42}+12 c_{4 \square}^{2}+2 c_{66}, \\
\alpha_{2}^{z H \bar{H}} & =-\frac{37}{4} c_{4 \square}^{2}-c_{66}, \\
\alpha_{1}^{z R} & =\frac{1}{8} c_{4 \square}^{2}-\frac{3}{2} c_{66}, \\
\alpha_{2}^{\bar{G}} & =c_{45}-2 c_{4} c_{4 \square}-4 c_{4}^{\prime} c_{4 \square}-4 c_{4 \square}^{2}+12 c_{66}, \\
\alpha_{3}^{z K^{2}} & =-4 c_{45}+4 c_{4} c_{4 \square}+8 c_{4}^{\prime} c_{4 \square}+8 c_{4 \square}^{2}-32 c_{66}, \\
\alpha_{1}^{\bar{z} S} & =-\frac{23}{8} c_{4 \square}^{2}+\frac{5}{2} c_{66}, \\
\alpha_{2}^{z^{2} \bar{H} B} & =\frac{37}{8} c_{4 \square}^{2}+\frac{1}{2} c_{66}, \\
\alpha_{2}^{\bar{z}^{2} J} & =\frac{17}{2} c_{4 \square}^{2}-6 c_{66}, \\
\alpha_{3}^{\bar{z}^{3} H^{2}} & =\frac{5}{4} c_{4 \square}^{2}+c_{66}, \\
\alpha_{1}^{\bar{z} E} & =-\frac{3}{2} c_{4 \square}^{2}+2 c_{66}, \\
\beta_{3}^{z K^{2}} & =4 c_{24}+2 c_{53}, \\
\beta_{3}^{z H \bar{H}} & =-4 c_{4} c_{4 \square}+\frac{3}{2} c_{4 \square}^{2}+2 c_{53}-2 c_{66}, \\
\beta_{3}^{\bar{z} K H} & =-2 c_{45}+4 c_{4} c_{4 \square}+8 c_{4}^{\prime} c_{4 \square}+8 c_{4 \square}^{2}-4 c_{53}-16 c_{66}, \\
\beta_{2}^{H \bar{B}} & =2 c_{4} c_{4 \square}, \\
\beta_{1}^{H \bar{B}} & =-\frac{3}{4} c_{4 \square}^{2}+c_{66}, \\
\beta_{1}^{z B \bar{B}} & =\frac{3}{8} c_{4 \square}^{2}-\frac{1}{2} c_{66}, \\
\beta_{3}^{K B} & =c_{45}-2 c_{4} c_{4 \square}-4 c_{4}^{\prime} c_{4 \square}-4 c_{4 \square}^{2}+8 c_{66} .
\end{aligned}
$$

Adding three further total derivatives

$$
\begin{array}{r}
\left(3 c_{4 \square}^{2}-4 c_{66}\right) \partial_{\mu}\left[|z|^{2} \frac{H \partial_{\nu} \bar{z} \partial^{\mu} \partial^{\nu} \bar{z}}{M^{4}}\right]-\left(c_{45}+4 c_{66}\right) \partial_{\mu}\left[\frac{K B \partial^{\mu} \bar{z}}{M^{4}}\right] \\
+\left(\frac{3}{2} c_{4 \square}^{2}+2 c_{66}\right) \partial_{\mu}\left[\frac{z^{2} \bar{H} \bar{B} \partial^{\mu} z}{M^{4}}\right],
\end{array}
$$

to the fourth order Lagrangian and we arrive at eq. (3.88). For an exhaustive list of the possibilities for $\psi_{2}^{\alpha}$ and $\psi_{2}^{\beta}$ we have considered, see appendix B, whereas for the exhaustive list of possibilities of total derivatives on the Riemann sphere coordinate, see appendix C. The proof is completed by direct albeit tedious computations.

Lemma 12 The $N L \sigma M$ with $\mathrm{O}(3)$, parity and Lorentz invariance having a single mass scale $\Lambda$ to order $\Lambda^{-4}$ (3.74) with Lagrangian components (3.75), (3.83) and (3.88), is not box-free, but $\left(\partial_{0}^{2} z \partial_{0}^{2} \bar{z}\right),\left(\partial_{0}^{2} z\right)^{2}$ and $\left(\partial_{0}^{2} \bar{z}\right)^{2}$ can be removed by a subsequent field redefinition

$$
z \rightarrow z+\frac{1}{\Lambda^{4}}\left[c_{53} \frac{H \bar{B}}{M^{2}}+c_{4}^{K F} \frac{K B}{M^{2}}\right]
$$


for which the theory becomes

$$
\begin{aligned}
& \mathcal{L}^{(4)}+\delta \mathcal{L}^{(4)}=c_{\{6,5\}}^{K^{3}}\left[\frac{K^{3}}{M^{\{6,5\}}}\right]+c_{6}^{K H \bar{H}}\left[\frac{K H \bar{H}}{M^{6}}\right]+c_{6}^{z^{2} K^{2} \bar{H}}\left[\frac{z^{2} K^{2} \bar{H}+\bar{z}^{2} K^{2} H}{M^{6}}\right] \\
& -2 c_{53}\left[\frac{z^{2} H \bar{H}^{2}+\bar{z}^{2} \bar{H} H^{2}}{M^{6}}\right] \\
& +c_{53}\left[\frac{H \bar{E}+\bar{H} E}{M^{4}}\right]+c_{4}^{K F}\left[\frac{K F}{M^{4}}\right]-4 c_{53}\left[\frac{\bar{z} H G+z \bar{H} \bar{G}}{M^{5}}\right]+c_{5}^{z K \bar{I}}\left[\frac{z K \bar{I}+\bar{z} K I}{M^{5}}\right] \\
& -c_{53}\left[\frac{H \bar{B}^{2}+\bar{H} B^{2}}{M^{4}}\right]-c_{4}^{K F}\left[\frac{K B \bar{B}}{M^{4}}\right]+2 c_{53}\left[\frac{\bar{z} H \bar{H} B+z H \bar{H} \bar{B}}{M^{5}}\right] \\
& +2 c_{4}^{K F}\left[\frac{\bar{z} K H \bar{B}+z K \bar{H} B}{M^{5}}\right] .
\end{aligned}
$$

Proof. The field redefinition (3.93), after dropping a boundary term, contributes

$$
-\left(\frac{\bar{B}}{M^{2}}-\frac{2 \bar{H} z}{M^{3}}\right)\left(c_{53} \frac{H \bar{B}}{M^{2}}+c_{4}^{K F} \frac{K B}{M^{2}}\right)-\left(\frac{B}{M^{2}}-\frac{2 H \bar{z}}{M^{3}}\right)\left(c_{53} \frac{\bar{H} B}{M^{2}}+c_{4}^{K F} \frac{K \bar{B}}{M^{2}}\right),
$$

to the Lagrangian, which results in eq. (3.94). Writing out the time and spatial derivatives of the fourth-order Lagrangian (3.94), we have

$$
\begin{aligned}
\mathcal{L}^{(4)}+\delta \mathcal{L}^{(4)}= & c_{\{6,5\}}^{K^{3}}\left[\frac{\left(-\left|z_{0}\right|+\left|z_{i}\right|^{2}\right)^{3}}{\left.M^{\{6,5\}}\right]}\right. \\
& +c_{6}^{K H} \bar{H}\left[\frac{\left(-\left|z_{0}\right|^{2}+\left|z_{i}\right|^{2}\right)\left(-z_{0}^{2}+z_{j}^{2}\right)\left(-\bar{z}_{0}^{2}+\bar{z}_{k}^{2}\right)}{M^{6}}\right] \\
& +c_{6}^{z^{2}} K^{2} \bar{H}\left[\frac{z^{2}\left(-\left|z_{0}\right|^{2}+\left|z_{i}\right|^{2}\right)^{2}\left(-\bar{z}_{0}^{2}+\bar{z}_{j}^{2}\right)+\text { c.c. }}{M^{6}}\right] \\
& -2 c_{53}\left[\frac{\left.z^{2}\left(-z_{0}^{2}+z_{i}^{2}\right)\left(-\bar{z}_{0}^{2}+\bar{z}_{j}^{2}\right)^{2}+\text { c.c. }\right]}{M^{6}}\right] \\
& +c_{53}\left[\frac{\left.\left(-z_{0}^{2}+z_{k}^{2}\right)\left(-2 \bar{z}_{0 i}^{2}+\bar{z}_{i j}^{2}+2 \bar{z}_{00} \bar{z}_{i i}-\bar{z}_{i i} \bar{z}_{j j}\right)+\text { c.c. }\right]}{M^{4}}\right] \\
& +c_{4}^{K F}\left[\frac{\left(-\left|z_{0}\right|^{2}+\left|z_{k}\right|^{2}\right)\left(-2\left|z_{0 i}\right|^{2}+\left|z_{i j}\right|^{2}+z_{00} \bar{z}_{i i}+\bar{z}_{00} z_{i i}-z_{i i} \bar{z}_{j j}\right)}{M^{4}}\right] \\
& -2 c_{53}\left[\frac{\bar{z}\left(-z_{0}^{2}+z_{k}^{2}\right)\left(\bar{z}_{0}^{2} z_{00}+\bar{z}_{i}^{2} z_{00}-4 \bar{z}_{0} \bar{z}_{i} z_{0 i}+2 \bar{z}_{i} \bar{z}_{j} z_{i j}+\bar{z}_{0}^{2} z_{i i}-\bar{z}_{i}^{2} z_{j j}\right)+\text { c.c. }}{M^{5}}\right] \\
& +c_{5}^{z K \bar{I}}\left[\frac{\left.z\left(-\left|z_{0}\right|^{2}+\left|z_{k}\right|^{2}\right)\left(\left|z_{0}\right|^{2} \bar{z}_{00}-z_{0} \bar{z}_{i} \bar{z}_{0 i}-z_{i} \bar{z}_{0} \bar{z}_{0 i}+z_{i} \bar{z}_{j} \bar{z}_{i j}\right)+\text { c.c. }\right]}{M^{5}}\right] \\
& +2 c_{4}^{K F}\left[\frac{\bar{z}\left(-\left|z_{0}\right|^{2}+\left|z_{i}\right|^{2}\right)\left(-z_{0}^{2}+z_{j}^{2}\right)\left(-\bar{z}_{00}+\bar{z}_{k k}\right)+\text { c.c. }}{M^{5}}\right]
\end{aligned}
$$


from which it is clear that all terms proportional to $z_{00}^{2}, \bar{z}_{00}^{2}$ and $\left|z_{00}\right|^{2}$ have been canceled out and we have used the short-hand notation $z_{\mu}=\partial_{\mu} z$, etc.

Remark 13 Notice that the last five lines of eq. (3.96) are linearly dependent on either $z_{00}$ or $\bar{z}_{00}$ (and not both of them). Technically, the assumption of non-degeneracy $\left(\frac{\partial^{2} \mathcal{L}}{\partial z_{00}^{2}} \neq 0\right)$ is violated. The Ostrogradsky theorem implying instability is thus not valid, but unfortunately, that is not necessarily sufficient for ruling out instability.

In order to illustrate the problem of a residual instability, let us consider a subLagrangian of the full theory (3.94), i.e.,

$$
\begin{aligned}
\mathcal{L} & =-2 \Lambda^{d-5} c_{4}^{K F} \frac{K(F-B \bar{B})}{M^{4}} \\
& =-\frac{K(F-B \bar{B})}{M^{4}} \\
& =-\frac{\left(-\left|z_{0}\right|^{2}+\left|z_{i}\right|^{2}\right)\left(-2\left|z_{0 i}\right|^{2}+\left|z_{i j}\right|^{2}+z_{00} \bar{z}_{i i}+\bar{z}_{00} z_{i i}-z_{i i} \bar{z}_{j j}\right)}{M^{4}},
\end{aligned}
$$

where on the second line, we have set the overall factor equal to unity and in the last line written out the time and spatial derivatives, explicitly. Defining now

$$
\begin{aligned}
\pi_{z}= & \frac{\partial \mathcal{L}}{\partial z_{0}}-\partial_{t}\left(\frac{\partial \mathcal{L}}{\partial z_{00}}\right) \\
= & \frac{\bar{z}_{0}\left(-2\left|z_{0 i}\right|^{2}+\left|z_{i j}\right|^{2}+z_{00} \bar{z}_{i i}+\bar{z}_{00} z_{i i}-z_{i i} \bar{z}_{j j}\right)}{M^{4}}+\frac{\left(-z_{00} \bar{z}_{0}-z_{0} \bar{z}_{00}+z_{0 i} \bar{z}_{i}+z_{i} \bar{z}_{0 i}\right) \bar{z}_{j j}}{M^{4}} \\
& -\frac{4 K \bar{z}_{i i}\left(z_{0} \bar{z}+z \bar{z}_{0}\right)}{M^{5}}, \\
\pi_{w}= & \frac{\partial \mathcal{L}}{\partial z_{00}}=-\frac{K \bar{z}_{i i}}{M^{4}}, \\
\pi_{z_{i}}= & \frac{\partial \mathcal{L}}{\partial z_{0 i}}=\frac{2 K \bar{z}_{0 i}}{M^{4}}, \\
w= & z_{0}, \\
w_{i}= & z_{0 i},
\end{aligned}
$$

we can write down the Ostrogradsky Hamiltonian

$$
\begin{aligned}
\mathcal{H}= & \pi_{z} z_{0}+\bar{\pi}_{z} \bar{z}_{0}+\pi_{w} z_{00}+\bar{\pi}_{w} \bar{z}_{00}+\pi_{z_{i}} z_{0 i}+\bar{\pi}_{z_{i}} \bar{z}_{0 i}-\mathcal{L} \\
= & \frac{\left|z_{0}\right|^{2}\left(-6\left|z_{0 i}\right|^{2}+\left|z_{i j}\right|^{2}+z_{00} \bar{z}_{i i}+\bar{z}_{00} z_{i i}-z_{i i} \bar{z}_{j j}\right)}{M^{4}} \\
& +\frac{\left(-z_{0}^{2} \bar{z}_{00}+z_{0 i} \bar{z}_{i} z_{0}+z_{i} \bar{z}_{0 i} z_{0}\right) \bar{z}_{j j}}{M^{4}}+\frac{\left(-\bar{z}_{0}^{2} z_{00}+\bar{z}_{0 i} z_{i} \bar{z}_{0}+\bar{z}_{i} z_{0 i} \bar{z}_{0}\right) z_{j j}}{M^{4}} \\
& -\frac{4 K \bar{z}_{i i}\left(z_{0}^{2} \bar{z}+z\left|z_{0}\right|^{2}\right)}{M^{5}}-\frac{4 K z_{i i}\left(\bar{z}_{0}^{2} z+\bar{z}\left|z_{0}\right|^{2}\right)}{M^{5}}+\frac{\left|z_{k}\right|^{2}\left(2\left|z_{0 i}\right|^{2}+\left|z_{i j}\right|^{2}-z_{i i} \bar{z}_{j j}\right)}{M^{4}},
\end{aligned}
$$

where the Legendre transform has canceled out the terms linear in $z_{00}$ and $\bar{z}_{00}$ between $\pi_{w} z_{00}+\bar{\pi}_{w} \bar{z}_{00}$ and $\mathcal{L}$. But, unfortunately, there remains linear dependence on $z_{00}$ and $\bar{z}_{00}$ inside $\pi_{z}$ which is not canceled out in the resulting Hamiltonian. Their presence in $\pi_{z}$ is 
indeed a necessity, since the third-order derivative present in the Euler-Lagrange equation of motion corresponding to the Lagrangian, manifests itself in the Hamilton equation as

$$
\partial_{t} \pi_{z}=-\frac{\partial \mathcal{H}}{\partial z}
$$

Writing now the Hamiltonian in terms of the phase-space variables $z, w, w_{i}, \pi_{z}, \pi_{w}, \pi_{z_{i}}$ and their complex conjugates, we have

$$
\mathcal{H}=\pi_{z} w+\bar{\pi}_{z} \bar{w}+\frac{1}{2} \pi_{z_{i}} w_{i}+\frac{1}{2} \bar{\pi}_{z_{i}} \bar{w}_{i}+\frac{\left(-|w|^{2}+\left|z_{k}\right|^{2}\right)\left(\left|z_{i j}\right|^{2} z_{i i} \bar{z}_{j j}\right)}{M^{4}} .
$$

Allowing for spatial derivatives of the phase-space variables, since this is a Hamiltonian density for a field theory, we can see that the Hamiltonian is indeed still linearly dependent on $\pi_{z}$ and its complex conjugate, and the Ostrogradsky instability is thus not avoided.

Lemma 14 A sufficient condition for avoiding the Ostrogradsky instability, defined by the presence of a linearly dependent conjugate momentum in the corresponding Ostrogradsky Hamiltonian, can be stated as follows: the conjugate momentum $\pi_{w}=\frac{\partial \mathcal{L}}{\partial z_{00}}$ cannot contain time derivatives of any fields other than $z$.

Proof. The trouble of generating terms linear in $z_{00}$ and $\bar{z}_{00}$ in the conjugate momentum $\pi_{z}$ can be traced back to the fact that the prefactor of the linear term in $z_{00}$ contains the time derivative of another field, i.e. $\bar{z}_{0}$ and not only $z_{0}$. That is, consider

$$
\mathcal{L}=c\left(z_{0}^{p} z_{00}+\bar{z}_{0}^{p} \bar{z}_{00}\right), \quad c \in \mathbb{R}, \quad p \in \mathbb{Z}_{+},
$$

then we have the corresponding conjugate momentum

$$
\pi_{z}=\frac{\partial \mathcal{L}}{\partial z_{0}}-\partial_{t}\left(\frac{\partial \mathcal{L}}{\partial z_{00}}\right)=c p z_{0}^{p-1} z_{00}-c p z_{0}^{p-1} z_{00}=0 .
$$

But consider instead another real term

$$
\mathcal{L}=c\left|z_{0}\right|^{2 p}\left(z_{00}+\bar{z}_{00}\right), \quad c \in \mathbb{R}, \quad p \in \mathbb{Z}_{+},
$$

then we have the conjugate momentum

$$
\begin{aligned}
\pi_{z}=\frac{\partial \mathcal{L}}{\partial z_{0}}-\partial_{t}\left(\frac{\partial \mathcal{L}}{\partial z_{00}}\right) & =c p\left|z_{0}\right|^{2(p-1)} \bar{z}_{0} z_{00}-c p\left|z_{0}\right|^{2(p-1)} \bar{z}_{0} z_{00}-c p\left|z_{0}\right|^{2(p-1)} z_{0} \bar{z}_{00} \\
& =-c p\left|z_{0}\right|^{2(p-1)} z_{0} \bar{z}_{00}
\end{aligned}
$$

and hence we have generated a term linear in $\bar{z}_{00}$ in $\pi_{z}$ which is not canceled by anything, as the Hamiltonian gets the induced terms

$$
\mathcal{H} \supset-c p\left|z_{0}\right|^{2(p-1)}\left(z_{0}^{2} \bar{z}_{00}+\bar{z}_{0}^{2} z_{00}\right)
$$

not present in the Lagrangian. Generalizing the proof to letting $\frac{\partial \mathcal{L}}{\partial z_{00}}$ contain functions of $z$ and $\bar{z}$ does not alter the conclusion and hence the Lemma follows. 
Corollary 15 Using Lemma 14, we can see that every term with linear dependence on $z_{00}$ in the Lagrangian (3.96) also has dependence on $\bar{z}_{0}$ and hence does not obey the condition of the Lemma to avoid the Ostrogradsky instability.

The problem of canceling off the terms linear in $z_{00}$ and $\bar{z}_{00}$ in the Lagrangian by using field redefinitions, is due to the metric on $\mathbb{C} P^{1}$ that generates always two terms, for example in the form proportional to $\frac{\bar{B}}{M^{2}}$ and $-\frac{2 \bar{H} z}{M^{3}}$. The stringency lies in keeping intact $\mathrm{O}(3)$ or $\mathbb{C} P^{1}$ symmetry as well as Lorentz symmetry. If we allow for relaxing the latter condition, we can make some progress on the problem.

Proposition 16 The $N L \sigma M$ with $\mathrm{O}(3)$, parity and Lorentz invariance having a single mass scale $\Lambda$ to order $\Lambda^{-4}$ (3.74) with Lagrangian components (3.75), (3.83) and (3.94), contains only terms linearly dependent on $\partial_{0}^{2} z$ or $\partial_{0}^{2} \bar{z}$, which can be removed by a subsequent field redefinition that includes Lorentz non-invariant terms

$$
z \rightarrow z+\frac{1}{\Lambda^{4}}\left[-c_{53} \frac{2 H \bar{z}_{i i}}{M^{2}}-c_{4}^{K F} \frac{K z_{i i}}{M^{2}}+2 c_{53} \frac{z \bar{H}\left(z_{0}^{2}+z_{i}^{2}\right)}{M^{3}}-c_{5}^{z K \bar{I}} \frac{z K\left|z_{0}\right|^{2}}{M^{3}}+2 c_{4}^{K F} \frac{\bar{z} K H}{M^{3}}\right]
$$

for which the theory becomes

$$
\begin{aligned}
& \mathcal{L}^{(4)}+\delta \mathcal{L}^{(4)}=c_{\{6,5\}}^{K^{3}}\left[\frac{K^{3}}{M^{\{6,5\}}}\right]+c_{6}^{K H \bar{H}}\left[\frac{K H \bar{H}}{M^{6}}\right]+c_{6}^{z^{2} K^{2} \bar{H}}\left[\frac{z^{2} K^{2} \bar{H}+\bar{z}^{2} K^{2} H}{M^{6}}\right] \\
& -2 c_{53}\left[\frac{z^{2} H \bar{H}^{2}+\bar{z}^{2} \bar{H} H^{2}}{M^{6}}\right]+4 c_{4}^{K F}\left[\frac{|z|^{2} K H \bar{H}}{M^{6}}\right] \\
& +c_{53}\left[\frac{H \bar{E}+\bar{H} E}{M^{4}}\right]+c_{4}^{K F}\left[\frac{K F}{M^{4}}\right]-4 c_{53}\left[\frac{\bar{z} H G+z \bar{H} \bar{G}}{M^{5}}\right]+c_{5}^{z K \bar{I}}\left[\frac{z K \bar{I}+\bar{z} K I}{M^{5}}\right] \\
& -c_{53}\left[\frac{H \bar{B}^{2}+\bar{H} B^{2}}{M^{4}}\right]-c_{4}^{K F}\left[\frac{K B \bar{B}}{M^{4}}\right]+2 c_{53}\left[\frac{\bar{z} H \bar{H} B+z H \bar{H} \bar{B}}{M^{5}}\right] \\
& +2 c_{53}\left[\frac{H \bar{B} \bar{z}_{i i}+\bar{H} B z_{i i}}{M^{4}}\right]+c_{4}^{K F}\left[\frac{K \bar{B} z_{i i}+K B \bar{z}_{i i}}{M^{4}}\right] \\
& -2 c_{53}\left[\frac{z \bar{H} \bar{B}\left(z_{0}^{2}+z_{i}^{2}\right)+\bar{z} H B\left(\bar{z}_{0}^{2}+\bar{z}_{i}^{2}\right)}{M^{5}}\right]+c_{5}^{z K \bar{I}}\left[\frac{z K \bar{B}\left|z_{0}\right|^{2}+\bar{z} K B\left|z_{0}\right|^{2}}{M^{5}}\right] \\
& -4 c_{53}\left[\frac{z H \bar{H} \bar{z}_{i i}+\bar{z} H \bar{H} z_{i i}}{M^{5}}\right]-c_{4}^{K F}\left[\frac{z K \bar{H} z_{i i}+\bar{z} K H \bar{z}_{i i}}{M^{5}}\right] \\
& +2 c_{53}\left[\frac{z^{2} \bar{H}^{2}\left(z_{0}^{2}+z_{i}^{2}\right)+\bar{z}^{2} H^{2}\left(\bar{z}_{0}^{2}+\bar{z}_{i}^{2}\right)}{M^{6}}\right]-c_{5}^{z K \bar{I}}\left[\frac{z^{2} K \bar{H}\left|z_{0}\right|^{2}+\bar{z}^{2} K H\left|z_{0}\right|^{2}}{M^{6}}\right] .
\end{aligned}
$$


Proof. The field redefinition (3.111), after dropping boundary terms, contributes

$$
\begin{aligned}
& \left(\frac{\bar{B}}{M^{2}}-\frac{2 \bar{H} z}{M^{3}}\right)\left(c_{53} \frac{2 H \bar{z}_{i i}}{M^{2}}+c_{4}^{K F} \frac{K z_{i i}}{M^{2}}-2 c_{53} \frac{z \bar{H}\left(z_{0}^{2}+z_{i}^{2}\right)}{M^{3}}+c_{5}^{z K \bar{I}} \frac{z K\left|z_{0}\right|^{2}}{M^{3}}-2 c_{4}^{K F} \frac{\bar{z} K H}{M^{3}}\right) \\
& +\left(\frac{B}{M^{2}}-\frac{2 H \bar{z}}{M^{3}}\right)\left(c_{53} \frac{2 H \bar{z}_{i i}}{M^{2}}+c_{4}^{K F} \frac{K z_{i i}}{M^{2}}-2 c_{53} \frac{z \bar{H}\left(z_{0}^{2}+z_{i}^{2}\right)}{M^{3}}+c_{5}^{z K \bar{I}} \frac{z K\left|z_{0}\right|^{2}}{M^{3}}-2 c_{4}^{K F} \frac{\bar{z} K H}{M^{3}}\right),
\end{aligned}
$$

to the Lagrangian, which results in eq. (3.112). Writing out the time and spatial derivatives of the fourth-order Lagrangian (3.112), we have

$$
\begin{aligned}
& \mathcal{L}^{(4)}+\delta \mathcal{L}^{(4)}=c_{\{6,5\}}^{K^{3}}\left[\frac{K^{3}}{M^{\{6,5\}}}\right]+c_{6}^{K H} \bar{H}\left[\frac{K H \bar{H}}{M^{6}}\right]+c_{6}^{z^{2} K^{2} \bar{H}}\left[\frac{z^{2} K^{2} \bar{H}+\bar{z}^{2} K^{2} H}{M^{6}}\right] \\
& -2 c_{53}\left[\frac{z^{2} H \bar{H}^{2}+\bar{z}^{2} \bar{H} H^{2}}{M^{6}}\right]+4 c_{4}^{K F}\left[\frac{|z|^{2} K H \bar{H}}{M^{6}}\right]+c_{53}\left[\frac{H\left(-2 \bar{z}_{0 i}^{2}+\bar{z}_{i j}^{2}+\bar{z}_{i i} \bar{z}_{j j}\right)+\text { c.c. }}{M^{4}}\right] \\
& +c_{4}^{K F}\left[\frac{K\left(-2\left|z_{0 i}\right|^{2}+\left|z_{i j}\right|^{2}+z_{i i} \bar{z}_{j j}\right)}{M^{4}}\right] \\
& -2 c_{53}\left[\frac{\bar{z} H\left(-4 \bar{z}_{0} \bar{z}_{i} z_{0 i}+2 \bar{z}_{i} \bar{z}_{j} z_{i j}+2 \bar{z}_{i}^{2} z_{j j}\right)+\text { c.c. }}{M^{5}}\right] \\
& +c_{5}^{z K \bar{I}}\left[\frac{z K\left(-z_{0} \bar{z}_{i} \bar{z}_{0 i}-z_{i} \bar{z}_{0} \bar{z}_{0 i}+z_{i} \bar{z}_{j} \bar{z}_{i j}+\left|z_{0}\right|^{2} z_{i i}\right)+\text { c.c. }}{M^{5}}\right]-c_{4}^{K F}\left[\frac{z K \bar{H} z_{i i}+\text { c.c. }}{M^{5}}\right] \\
& +2 c_{53}\left[\frac{z^{2} \bar{H}^{2}\left(z_{0}^{2}+z_{i}^{2}\right)+\bar{z}^{2} H^{2}\left(\bar{z}_{0}^{2}+\bar{z}_{i}^{2}\right)}{M^{6}}\right]-c_{5}^{z K \bar{I}}\left[\frac{z^{2} K \bar{H}\left|z_{0}\right|^{2}+\text { c.c. }}{M^{6}}\right] \text {. }
\end{aligned}
$$

The above Lagrangian contains no $\partial_{0}^{2} z$ or $\partial_{0}^{2} \bar{z}$ terms and hence has a second-order equation of motion and thus no Ostrogradsky instability, but it does not enjoy Lorentz invariance.

Remark 17 The Lagrangian (3.114) is manifestly free from double time derivatives on any single fields, at the cost of lost Lorentz invariance. The spatial part of the Lorentz group $\mathrm{SO}(d) \subset \mathrm{SO}(d, 1)$ is unbroken, however, and a physical interpretation of the theory as a low-energy EFT (effective field theory), is that the rest frame is a preferred frame.

Remark 18 Our results are consistent with those of ref. [41], which however utilizes the equations of motion to eliminate the higher-order time derivatives from the EFT. Normally, it is not allowed to modify the Lagrangian using the equations of motion (e.g. the Dirac Lagrangian would disappear), but it is claimed [41] that for EFTs, there exists a field redefinition (or canonical transformation) that is equivalent to using the equations of motion, to first order in a given coupling constant. We do not rely on such assumption or perturbativity (apart from the derivative expansion itself), and work directly with field redefinitions. Whether our theory non-manifestly possesses (hidden) Lorentz invariance or not, is an open problem that we will leave for future studies.

Remark 19 Although the Lagrangian (3.114) manifestly avoids the Ostrogradsky instability, it is not yet manifestly free from runaway instabilities due to the nonlinearities in the first-order time derivatives. 
In order to contemplate the stability of the energy corresponding to the Lagrangian (3.114), we will calculate its corresponding Hamiltonian.

Lemma 20 The $N L \sigma M$ with $\mathrm{O}(3)$, parity and Lorentz invariance having a single mass scale $\Lambda$ to order $\Lambda^{-4}$ (3.74) with Lagrangian components (3.75), (3.83) and (3.112), has a corresponding Hamiltonian given by

$$
\mathcal{H}=2 \Lambda^{d-1}\left\{\mathcal{H}^{(0)}+\frac{1}{\Lambda^{2}} \mathcal{H}^{(2)}+\frac{1}{\Lambda^{4}} \mathcal{H}^{(4)}+\mathcal{O}\left(\Lambda^{-6}\right)\right\},
$$

with

$$
\begin{aligned}
& \mathcal{H}^{(0)}=\frac{\widetilde{K}}{M^{2}}, \\
& \mathcal{H}^{(2)}=\left(2 c_{4}+4 c_{4}^{\prime}+4 c_{4 \square}\right) \frac{K\left(3\left|z_{0}\right|^{2}+\left|z_{j}\right|^{2}\right)}{M^{4}}-2 c_{4} \frac{-3\left|z_{0}\right|^{4}+z_{0}^{2} \bar{z}_{i}^{2}+\bar{z}_{0}^{2} z_{i}^{2}+z_{i}^{2} \bar{z}_{j}^{2}}{M^{4}}, \\
& \mathcal{H}^{(4)}=c_{\{6,5\}}^{K^{3}}\left[\frac{K^{2}\left(5\left|z_{0}\right|^{2}+\left|z_{i}\right|^{2}\right)}{M^{\{6,5\}}}\right]+c_{6}^{K H} \bar{H}\left[\frac{\widetilde{K} H \bar{H}+2 K H \bar{z}_{0}^{2}+2 K \bar{H} z_{0}^{2}}{M^{6}}\right] \\
& +c_{6}^{z^{2} K^{2} \bar{H}}\left[\frac{z^{2} K \bar{H}\left(3\left|z_{0}\right|^{2}+\left|z_{i}\right|^{2}\right)+2 z^{2} K^{2} \bar{z}_{0}^{2}+\text { c.c. }}{M^{6}}\right] \\
& -2 c_{53}\left[\frac{z^{2} \bar{H}^{2} \widetilde{H}+4 z^{2} H \bar{H} z_{0}^{2}+c . c .}{M^{6}}\right]+4 c_{4}^{K F}\left[\frac{|z|^{2}\left(\widetilde{K} H \bar{H}+2 K H \bar{z}_{0}^{2}+2 K \bar{H} z_{0}^{2}\right)}{M^{6}}\right] \\
& +c_{53}\left[\frac{\widetilde{H}\left(\bar{z}_{i j}^{2}+\bar{z}_{i i} \bar{z}_{j j}\right)+2\left(-3 z_{0}^{2}+z_{i}^{2}\right) \bar{z}_{0 j}^{2}+\text { c.c. }}{M^{4}}\right] \\
& +c_{4}^{K F}\left[\frac{\widetilde{K}\left(\left|z_{i j}\right|^{2}+z_{i i} \bar{z}_{j j}\right)+2\left(-3\left|z_{0}\right|^{2}+\left|z_{i}\right|^{2}\right)\left|z_{0 j}\right|^{2}}{M^{4}}\right] \\
& -2 c_{53}\left[\frac{2 \bar{z} \widetilde{H}\left(\bar{z}_{i} \bar{z}_{j} z_{i j}+\bar{z}_{i}^{2} z_{j j}\right)+4 \bar{z}\left(-3 z_{0}^{2}+z_{i}^{2}\right) \bar{z}_{0} \bar{z}_{j} z_{0 j}+\text { c.c. }}{M^{5}}\right] \\
& +c_{5}^{z K \bar{I}}\left[\frac{z \widetilde{K} z_{i} \bar{z}_{j} \bar{z}_{i j}-z\left(-3\left|z_{0}\right|^{2}+\left|z_{i}\right|^{2}\right)\left(-z_{0} \bar{z}_{i} \bar{z}_{0 i}-z_{i} \bar{z}_{0} \bar{z}_{0 i}+\left|z_{0}\right|^{2} z_{i i}\right)+c . c .}{M^{5}}\right] \\
& -c_{4}^{K F}\left[\frac{z \widetilde{K} \bar{H} z_{i i}+2 z \bar{z}_{0}^{2} K z_{i i}+\text { c.c. }}{M^{5}}\right] \\
& +2 c_{53}\left[\frac{\bar{z}^{2}\left(3 z_{0}^{2}+z_{i}^{2}\right) H\left(\bar{z}_{0}^{2}+\bar{z}_{i}^{2}\right)-2 \bar{z}^{2} \bar{z}_{0}^{2} H^{2}+\text { c.c. }}{M^{6}}\right] \\
& -c_{5}^{z K \bar{I}}\left[\frac{\bar{z}^{2}\left(3\left|z_{0}\right|^{2}-\left|z_{i}\right|^{2}\right) H\left|z_{0}\right|^{2}+2 z_{0}^{2} \bar{z}^{2} K\left|z_{0}\right|^{2}+\text { c.c. }}{M^{6}}\right],
\end{aligned}
$$

and the definitions

$$
\widetilde{K}=\left|z_{0}\right|^{2}+\left|z_{i}\right|^{2}, \quad \widetilde{H}=z_{0}^{2}+z_{i}^{2}, \quad \widetilde{\bar{H}}=\bar{z}_{0}^{2}+\bar{z}_{i}^{2} .
$$


Proof. Defining

$$
\begin{aligned}
\pi_{z} & =\frac{\partial \mathcal{L}}{\partial z_{0}}=-2 \Lambda^{d-1}\left\{\pi_{z}^{(0)}+\frac{1}{\Lambda^{2}} \pi_{z}^{(2)}+\frac{1}{\Lambda^{4}} \pi_{z}^{(4)}+\mathcal{O}\left(\Lambda^{-6}\right)\right\} \\
\pi_{z_{i}}=\frac{\partial \mathcal{L}}{\partial z_{0 i}} & =-2 \Lambda^{d-1}\left\{\frac{1}{\Lambda^{4}} \pi_{z_{i}}^{(4)}+\mathcal{O}\left(\Lambda^{-6}\right)\right\}
\end{aligned}
$$

the Hamiltonian is written via the Legendre transform as

$$
\mathcal{H}=\pi_{z} z_{0}+\bar{\pi}_{z} \bar{z}_{0}+\pi_{z_{i}} z_{0 i}+\bar{\pi}_{z_{i}} \bar{z}_{0 i}-\mathcal{L},
$$

and a long but straightforward calculation yields

$$
\begin{aligned}
& \pi_{z}^{(0)}=-\frac{\bar{z}_{0}}{M^{2}} \\
& \pi_{z}^{(2)}=-\left(2 c_{4}+4 c_{4}^{\prime}+4 c_{4 \square}\right) \frac{2 K \bar{z}_{0}}{M^{4}}+2 c_{4} \frac{2 \bar{H} z_{0}}{M^{4}}, \\
& \pi_{z}^{(4)}=-c_{\{6,5\}}^{K^{3}} \frac{3 K^{2} \bar{z}_{0}}{M^{\{6,5\}}}-c_{6}^{K H} \frac{\left(\bar{z}_{0} H+2 z_{0} K\right) \bar{H}}{M^{6}}-c_{6}^{z^{2} K^{2}} \bar{H}^{2} \frac{2 \bar{z}_{0}\left(z^{2} K \bar{H}+\bar{z}^{2} K H\right)+2 z_{0} \bar{z}^{2} K^{2}}{M^{6}} \\
& +2 c_{53} \frac{2 z_{0}\left(z^{2} \bar{H}^{2}+2 \bar{z}^{2} H \bar{H}\right)}{M^{6}}-4 c_{4}^{K F} \frac{|z|^{2}\left(\bar{z}_{0} H+2 z_{0} K\right) \bar{H}}{M^{6}} \\
& -c_{53} \frac{2 z_{0}\left(-2 \bar{z}_{0 i}^{2}+\bar{z}_{i j}^{2}+\bar{z}_{i i} \bar{z}_{j j}\right)}{M^{4}}-c_{4}^{K F} \frac{\bar{z}_{0}\left(-2\left|z_{0 i}\right|^{2}+\left|z_{i j}\right|^{2}+z_{i i} \bar{z}_{j j}\right)}{M^{4}} \\
& +2 c_{53} \frac{2 z_{0} \bar{z}\left(-4 \bar{z}_{0} \bar{z}_{i} z_{0 i}+2 \bar{z}_{i} \bar{z}_{j} z_{i j}+2 \bar{z}_{i}^{2} z_{j j}\right)+4 z \bar{H} z_{i} \bar{z}_{0 i}}{M^{5}} \\
& -c_{5}^{z K \bar{I}} \frac{\bar{z}_{0} z\left(-z_{0} \bar{z}_{i} \bar{z}_{0 i}-z_{i} \bar{z}_{0} \bar{z}_{0 i}+z_{i} \bar{z}_{j} \bar{z}_{i j}+\left|z_{0}\right|^{2} z_{i i}\right)}{M^{5}} \\
& -c_{5}^{z K \bar{I}} \frac{\bar{z}_{0} \bar{z}\left(-\bar{z}_{0} z_{i} z_{0 i}-\bar{z}_{i} z_{0} z_{0 i}+\bar{z}_{i} z_{j} z_{i j}+\left|z_{0}\right|^{2} \bar{z}_{i i}\right)-z K\left(-\bar{z}_{i} \bar{z}_{0 i}-\bar{z}_{i} z_{0 i}+\bar{z}_{0} z_{i i}+\bar{z}_{0} \bar{z}_{i i}\right)}{M^{5}} \\
& +c_{4}^{K F} \frac{\bar{z}_{0} z \bar{H} z_{i i}+\bar{z}_{0} \bar{z} H \bar{z}_{i i}+2 z_{0} \bar{z} K \bar{z}_{i i}}{M^{5}}-2 c_{53} \frac{4 z_{0} \bar{z}^{2} H\left(z_{0}^{2}+z_{i}^{2}\right)-2 z^{2} \bar{H}^{2} z_{0}}{M^{6}} \\
& +c_{5}^{z K \bar{I}} \frac{\bar{z}_{0} z^{2} \bar{H}\left|z_{0}\right|^{2}+\bar{z}_{0} \bar{z}^{2} H\left|z_{0}\right|^{2}-z^{2} K \bar{H} \bar{z}_{0}-\bar{z}^{2} K H \bar{z}_{0}}{M^{6}}, \\
& \pi_{z_{i}}^{(4)}=-c_{53} \frac{4 \bar{H} z_{0 i}}{M^{4}}-c_{4}^{K F} \frac{2 K \bar{z}_{0 i}}{M^{4}}+2 c_{53} \frac{4 \bar{z} H \bar{z}_{0} \bar{z}_{i}}{M^{5}}-c_{5}^{z K \bar{I}} \frac{\bar{z} K\left(-\bar{z}_{0} z_{i}-\bar{z}_{i} z_{0}\right)}{M^{5}} .
\end{aligned}
$$

A direct calculation yields the Hamiltonian given in eq. (3.118).

Theorem 21 The $N L \sigma M$ with $\mathrm{O}(3)$, parity and Lorentz invariance having a single mass scale $\Lambda$ to order $\Lambda^{-4}$ (3.74) with Lagrangian components (3.75), (3.83) and (3.112), is not bounded from below in the limit of large time derivatives, and the theory potentially suffers from a spiral instability or a runaway instability.

Proof. We start by taking the limit

$$
\lim _{\left|z_{i}\right| \rightarrow 0} \mathcal{H}^{(4)}=A \frac{5\left|z_{0}\right|^{6}}{M^{6}}+B \frac{5\left|z_{0}\right|^{4}\left(z^{2} \bar{z}_{0}^{2}+\bar{z}^{2} z_{0}^{2}\right)}{M^{6}}
$$


corresponding to large time derivatives (and negligible spatial derivatives) and we have used that $c_{5}^{K^{3}}+4 c_{4}^{K F}=0$ and defined

$$
A=c_{6}^{K^{3}}+c_{6}^{K H \bar{H}}-4 c_{4}^{K F}, \quad B=c_{6}^{z^{2} K^{2} \bar{H}}-4 c_{53}+c_{5}^{z K \bar{I}} .
$$

Writing $z=\rho e^{\mathrm{i} \theta}$, we have

$$
z_{0}=\left(\rho_{0}+\mathrm{i} \theta_{0} \rho\right) e^{\mathrm{i} \theta}
$$

and hence

$$
\begin{aligned}
\left|z_{0}\right|^{6} & =\left(\rho_{0}^{2}+\rho^{2} \theta_{0}^{2}\right)^{3}, \\
\left|z_{0}\right|^{4}\left(z^{2} \bar{z}_{0}^{2}+\bar{z}^{2} z_{0}^{2}\right) & =\left(\rho_{0}^{2}+\rho^{2} \theta_{0}^{2}\right)^{2} 2 \rho^{2}\left(\rho_{0}^{2}-\rho^{2} \theta_{0}^{2}\right) .
\end{aligned}
$$

We assume that $A>0$ is strictly positive, which is equal to the condition

$$
4 c_{11}+4 c_{12}+4 c_{13}+4 c_{23}+6 c_{24}+4 c_{34}-c_{45}>0 .
$$

The spiral instability can occur if $B>0$ and

$$
A<2 B \rho^{2},
$$

since in this case the coefficient of $\theta_{0}^{6}$ is negative and a fast rotating phase can lower the energy indefinitely.

On the other hand, we may tune the theory so as to have $B<0$, for which a runaway instability can occur if

$$
A<2|B| \rho^{2} .
$$

In either case, the critical modulus of the field is

$$
\rho^{\text {crit }}=\left|\frac{A}{2 B}\right|=\left|\frac{4 c_{11}+4 c_{12}+4 c_{13}+4 c_{23}+6 c_{24}+4 c_{34}-c_{45}}{-2 c_{24}+c_{45}+6 c_{53}+4 c_{66}}\right|,
$$

which when reached can drive the theory into lower and lower energies.

Remark 22 The unveiled instability can be avoided if $B=0$, which however would require a precise fine-tuning of theory, which is not expected generically to be the case.

Remark 23 The instability found in Theorem 21 is due to the nature of the derivative expansion of the effective low-energy theory and is a classical instability. The quantum considerations are beyond the scope of this paper. The instability of spiral type is mathematically different from the instability taking place at order $\Lambda^{-2}$, which however is always of the isotropic type.

\section{Acknowledgments}

We thank Lorenzo Bartolini, Johan Bijnens and Chris Halcrow for useful discussions. S. B. G. thanks the Outstanding Talent Program of Henan University for partial support. The work of S. B. G. is supported by the National Natural Science Foundation of China (Grants No. 11675223 and No. 12071111). The work of M.N. is supported in part by JSPS Grant-in-Aid for Scientific Research (KAKENHI Grant No. 18H01217). 


\section{A Ostrogradsky's theorem}

For convenience, we review the Ostrogradsky's theorem, specialized to second-order derivatives:

Theorem 24 (Ostrogradsky [7], Woodard [8]) Given a Lagrangian theory with quadratic and non-degenerate dependence on a second-order time derivative of a field, the corresponding Ostrogradsky Hamiltonian possesses a linear dependence on one of the two conjugate momenta, and resultantly the corresponding energy is not bounded neither from below nor from above.

Proof. Consider the Lagrangian $L\left(z, z_{0}, z_{00}\right)$ which is a functional of $z$, as well as its first and second-order time derivatives denoted by one and two zeros as indices, respectively, and the corresponding Ostrogradsky Hamiltonian $H\left(z, w, \pi_{z}, \pi_{w}\right)$ which is a functional of $z, w=z_{0}$ and their conjugate momenta $\pi_{z}$ and $\pi_{w} \cdot{ }^{2}$ The condition that the Lagrangian depends non-degenerately on $z_{00}$ means that

$$
\frac{\partial^{2} L}{\partial z_{00}^{2}} \neq 0
$$

Then the Hamiltonian is defined as

$$
H=\pi_{z} z_{0}+\bar{\pi}_{z} \bar{z}_{0}+\pi_{w} w_{0}+\bar{\pi}_{w} \bar{w}_{0}-L
$$

the conjugate momenta are given by

$$
\pi_{z}=\frac{\partial L}{\partial z_{0}}-\partial_{t}\left(\frac{\partial L}{\partial z_{00}}\right), \quad \pi_{w}=\frac{\partial L}{\partial z_{00}}
$$

and the Hamilton's equations are

$$
\begin{array}{ll}
\frac{\partial H}{\partial \pi_{z}}=z_{0}=w, & \frac{\partial H}{\partial z}=-\partial_{t} \pi_{z} \\
\frac{\partial H}{\partial \pi_{w}}=w_{0}, & \frac{\partial H}{\partial w}=-\partial_{t} \pi_{w} .
\end{array}
$$

Finally, the Hamiltonian can be written as

$$
H=\pi_{z} w+\bar{\pi}_{z} \bar{w}+\pi_{w} a\left(z, w, \pi_{w}\right)+\bar{\pi}_{w} \overline{a\left(z, w, \pi_{w}\right)}-L\left(z, w, a\left(z, w, \pi_{w}\right)\right),
$$

where the acceleration $a\left(z, w, \pi_{w}\right)$ is defined by

$$
\left.\frac{\partial L}{\partial z_{00}}\right|_{z_{0}=w, z_{00}=a}=\pi_{w}
$$

The Hamilton equations $z_{0}=\frac{\partial H}{\partial \pi_{z}}, w_{0}=\frac{\partial H}{\partial \pi_{w}}, \partial_{t} \pi_{w}=-\frac{\partial H}{\partial w}$ simply reproduce the phase space transformation (A.4)-(A.5), whereas $\partial_{t} \pi_{z}=-\frac{\partial H}{\partial z}$ reproduces the Euler-Lagrange

\footnotetext{
${ }^{2}$ Here we will use the notation that the functional dependence on $z$ includes the dependence on $\bar{z}$ and will not state so explicitly in order not to clutter the notation.
} 
equation of $L$. The assumption of non-degeneracy (A.1) implies that the phase space transformation (A.4)-(A.5) can be inverted to solve for $z_{00}$ in terms of $z, w$ and $\pi_{w}$, by means of the implicit function theorem, which is the statement (A.7). Crucially, the acceleration $a\left(z, w, \pi_{w}\right)$ does not depend on the conjugate momentum $\pi_{z}$, which is only needed for the third-order time derivative of $z$. Notice that the third-order time derivative only appears when the assumption of non-degeneracy (A.1) holds true.

Finally, we have that the Ostrogradsky Hamiltonian (A.6) depends only linearly on the conjugate momentum $\pi_{z}$. The linear dependence implies the Ostrogradsky instability, since the system can linearly be driven to lower and lower (or higher and higher) energies. This completes the proof.

\section{A.1 Example}

As a simple example, let us consider an extension of the Harmonic oscillator as

$$
L=-\frac{1}{2} \alpha z_{00}^{2}+\frac{1}{2} \beta z_{0}^{2}-\frac{1}{2} \gamma z^{2},
$$

with $z \in \mathbb{R}$ being a real field (or function of time), $\alpha>0, \beta>0, \gamma>0$ and the indices denote time derivatives. First we have

$$
w=z_{0}, \quad \pi_{z}=\beta z_{0}+\alpha z_{000}, \quad \pi_{w}=-\alpha z_{00},
$$

whereas the Ostrogradsky Hamiltonian reads

$$
\begin{aligned}
H & =\pi_{z} w+\pi_{w} w_{0}-L \\
& =\pi_{z} w-\frac{\pi_{w}^{2}}{2 \alpha}-\frac{\beta w^{2}}{2}+\frac{\gamma z^{2}}{2},
\end{aligned}
$$

where the linear dependence on $\pi_{z}$ is manifest. Inserting the explicit expression (A.9) for $\pi_{z}$

$$
H=\frac{\beta w^{2}}{2}+\alpha w z_{000}-\frac{\pi_{w}^{2}}{2 \alpha}+\frac{\gamma z^{2}}{2},
$$

the kinetic energy for $z$, i.e. $w^{2}$ is manifestly positive. Integrating by parts and dropping the boundary term, we can write

$$
H=\frac{\beta w^{2}}{2}-\frac{3 \pi_{w}^{2}}{2 \alpha}+\frac{\gamma z^{2}}{2},
$$

from which the terminology Ostrogradsky's ghost comes from; that is, the kinetic energy, $\pi_{w}^{2}$ is negative semi-definite.

\section{B Field redefinition operators}

In order to cancel the unwanted operators, we need a systematic educated guess for the field redefinitions, $\psi_{2}$, at the order $\Lambda^{-4}$ and they read

$$
\begin{aligned}
& \frac{z K^{2}}{M^{\#}}, \frac{\bar{z} K H}{M^{\#}}, \frac{z^{3} K \bar{H}}{M^{\#}}, \frac{K B}{M^{\#}}, \frac{z^{2} K \bar{B}}{M^{\#}}, \frac{z H \bar{H}}{M^{\#}}, \frac{\bar{z}^{3} H^{2}}{M^{\#}}, \frac{z^{5} \bar{H}^{2}}{M^{\#}}, \frac{\bar{z}^{2} H B}{M^{\#}}, \frac{H \bar{B}}{M^{\#}}, \frac{z^{2} \bar{H} B}{M^{\#}}, \\
& \frac{z^{4} \bar{H} \bar{B}}{M^{\#}}, \frac{z B \bar{B}}{M^{\#}}, \frac{\bar{z} B^{2}}{M^{\#}}, \frac{z^{3} \bar{B}^{2}}{M^{\#}}, \frac{\bar{z} E}{M^{\#}}, \frac{z^{3} \bar{E}}{M^{\#}}, \frac{z F}{M^{\#}}, \frac{z^{2} G}{M^{\#}}, \frac{\bar{G}}{M^{\#}}, \frac{I}{M^{\#}}, \frac{z^{2} \bar{I}}{M^{\#}}, \frac{\bar{z}^{2} J}{M^{\#}}, \frac{z^{4} \bar{J}^{2}}{M^{\#}}, \\
& \frac{z R}{M^{\#}}, \frac{z \bar{R}}{M^{\#}}, \frac{\bar{z} S}{M^{\#}}, \frac{z^{3} \bar{S}}{M^{\#}},
\end{aligned}
$$


which is the complete list of four derivative operators with one factor of $z$ more than factors of $\bar{z}$ (with "chirality" +1 ), needed for generating sixth-order derivative terms by field redefinitions according to eq. (3.80), where $M^{\#}$ includes a term for each integer power necessary.

Notice that additional factors of $|z|^{2},|z|^{4}$ are already included in the above list by using that $|z|^{2}=M-1$ and hence an operator, e.g.,

$$
\frac{|z|^{2} X}{M^{n}}=-\frac{X}{M^{n}}+\frac{X}{M^{n-1}},
$$

and since all possible powers of $1 / M$ are included, such possibilities are already taken into account.

\section{Total derivatives}

In order to cancel unwanted operators, we also need a systematic educated guess for writing down total derivative operators that can be added to the Lagrangian; with six derivatives and four fields

$$
\begin{aligned}
& \partial_{\mu}\left(\frac{\bar{H} z^{\mu \nu} z_{\nu}+\text { c.c. }}{M^{\#}}\right), \partial_{\mu}\left(\frac{\bar{z} z^{\mu \nu} z_{\nu \rho} \bar{z}^{\rho}+\text { c.c. }}{M^{\#}}\right), \partial_{\mu}\left(\frac{\bar{z} \bar{B} z^{\mu \nu} z_{\nu}+\text { c.c. }}{M^{\#}}\right), \\
& \partial_{\mu}\left(\frac{\bar{z} z^{\mu \nu} \bar{z}_{\nu \rho} z^{\rho}+\text { c.c. }}{M^{\#}}\right), \partial_{\mu}\left(\frac{\bar{z} B z^{\mu \nu} \bar{z}_{\nu}+\text { c.c. }}{M^{\#}}\right), \partial_{\mu}\left(\frac{z \bar{B} z^{\mu \nu} \bar{z}_{\nu}+\text { c.c. }}{M^{\#}}\right), \\
& \partial_{\mu}\left(\frac{K z^{\mu \nu} \bar{z}_{\nu}+\text { c.c. }}{M^{\#}}\right), \partial_{\mu}\left(\frac{z z^{\mu \nu} \bar{z}_{\nu \rho} \bar{z}^{\rho}+\text { c.c. }}{M^{\#}}\right), \partial_{\mu}\left(\frac{\bar{I} z^{\mu}+\text { c.c. }}{M^{\#}}\right), \partial_{\mu}\left(\frac{\bar{H} B z^{\mu}+\text { c.c. }}{M^{\#}}\right), \\
& \partial_{\mu}\left(\frac{z \bar{B}^{2} z^{\mu}+\text { c.c. }}{M^{\#}}\right), \partial_{\mu}\left(\frac{\bar{z} B \bar{B} z^{\mu}+\text { c.c. }}{M^{\#}}\right), \partial_{\mu}\left(\frac{G z^{\mu}+\text { c.c. }}{M^{\#}}\right), \partial_{\mu}\left(\frac{K \bar{B} z^{\mu}+\text { c.c. }}{M^{\#}}\right) ;
\end{aligned}
$$

with six derivatives and six fields

$$
\begin{aligned}
& \partial_{\mu}\left(\frac{z^{2} \bar{H} z^{\mu \nu} \bar{z}_{\nu}+\text { c.c. }}{M^{\#}}\right), \partial_{\mu}\left(\frac{z^{2} \bar{H} \bar{B} z^{\mu}+\text { c.c. }}{M^{\#}}\right), \partial_{\mu}\left(\frac{z^{2} \bar{J} z^{\mu}+\text { c.c. }}{M^{\#}}\right), \\
& \partial_{\mu}\left(\frac{\bar{z}^{3} z^{\mu \nu} z_{\nu \rho} z^{\rho}+\text { c.c. }}{M^{\#}}\right), \partial_{\mu}\left(\frac{\bar{z}^{2} K z^{\mu \nu} z_{\nu}+\text { c.c. }}{M^{\#}}\right), \partial_{\mu}\left(\frac{\bar{z}^{2} H z^{\mu \nu} \bar{z}_{\nu}+\text { c.c. }}{M^{\#}}\right), \\
& \partial_{\mu}\left(\frac{\bar{z}^{2} I z^{\mu}+\text { c.c. }}{M^{\#}}\right), \partial_{\mu}\left(\frac{\bar{z}^{3} E z^{\mu}+\text { c.c. }}{M^{\#}}\right), \partial_{\mu}\left(\frac{\bar{z} H \bar{H} z^{\mu}+\text { c.c. }}{M^{\#}}\right), \partial_{\mu}\left(\frac{\bar{z}^{3} B^{2} z^{\mu}+\text { c.c. }}{M^{\#}}\right), \\
& \partial_{\mu}\left(\frac{z K \bar{H} z^{\mu}+\text { c.c. }}{M^{\#}}\right), \partial_{\mu}\left(\frac{\bar{z}^{2} H \bar{B} z^{\mu}+\text { c.c. }}{M^{\#}}\right), \partial_{\mu}\left(\frac{\bar{z} K^{2} z^{\mu}+\text { c.c. }}{M^{\#}}\right),
\end{aligned}
$$

are the complete lists of possibilities with no more than 3 derivatives acting on a single field. With only two fields, the total derivatives become trivial and we will not list them here.

Open Access. This article is distributed under the terms of the Creative Commons Attribution License (CC-BY 4.0), which permits any use, distribution and reproduction in any medium, provided the original author(s) and source are credited. 


\section{References}

[1] A.V. Manohar, Introduction to effective field theories, arXiv: 1804.05863 [INSPIRE].

[2] I. Brivio and M. Trott, The Standard Model as an effective field theory, Phys. Rept. 793 (2019) 1 [arXiv:1706.08945] [INSPIRE].

[3] S. Scherer, Introduction to chiral perturbation theory, Adv. Nucl. Phys. 27 (2003) 277 [hep-ph/0210398] [INSPIRE].

[4] E. Epelbaum, H.-W. Hammer and U.-G. Meissner, Modern theory of nuclear forces, Rev. Mod. Phys. 81 (2009) 1773 [arXiv:0811.1338] [inSPIRE].

[5] R. Machleidt and D.R. Entem, Chiral effective field theory and nuclear forces, Phys. Rept. 503 (2011) 1 [arXiv:1105.2919] [INSPIRE].

[6] S. Weinberg, Phenomenological lagrangians, Physica A 96 (1979) 327 [InSPIRE].

[7] M. Ostrogradsky, Mémoires sur les équations différentielles, relatives au problème des isopérimètres (in French), Mem. Acad. St. Petersbourg 6 (1850) 385 [InSPIRE].

[8] R.P. Woodard, Ostrogradsky's theorem on Hamiltonian instability, Scholarpedia 10 (2015) 32243 [arXiv: 1506.02210] [INSPIRE].

[9] A.R. Solomon and M. Trodden, Higher-derivative operators and effective field theory for general scalar-tensor theories, JCAP 02 (2018) 031 [arXiv:1709.09695] [INSPIRE].

[10] M. Asorey, F. Falceto and L. Rachwał, Asymptotic freedom and higher derivative gauge theories, JHEP 05 (2021) 075 [arXiv:2012.15693] [INSPIRE].

[11] A. Nicolis, R. Rattazzi and E. Trincherini, The Galileon as a local modification of gravity, Phys. Rev. D 79 (2009) 064036 [arXiv:0811.2197] [InSPIRE].

[12] G.W. Horndeski, Second-order scalar-tensor field equations in a four-dimensional space, Int. J. Theor. Phys. 10 (1974) 363 [InSPIRE].

[13] T.H.R. Skyrme, A nonlinear field theory, Proc. Roy. Soc. Lond. A 260 (1961) 127 [INSPIRE].

[14] S.B. Gudnason and M. Nitta, A higher-order Skyrme model, JHEP 09 (2017) 028 [arXiv: 1705.03438] [INSPIRE].

[15] L.D. Faddeev and A.J. Niemi, Partially dual variables in SU(2) Yang-Mills theory, Phys. Rev. Lett. 82 (1999) 1624 [hep-th/9807069] [INSPIRE].

[16] B.M.A.G. Piette, W.J. Zakrzewski, H.J.W. Mueller-Kirsten and D.H. Tchrakian, A modified Mottola-Wipf model with sphaleron and instanton fields, Phys. Lett. B 320 (1994) 294 [INSPIRE].

[17] B.M.A.G. Piette, B.J. Schroers and W.J. Zakrzewski, Multi-solitons in a two-dimensional Skyrme model, Z. Phys. C 65 (1995) 165 [hep-th/9406160] [INSPIRE].

[18] D. Anselmi and M. Piva, Perturbative unitarity of Lee-Wick quantum field theory, Phys. Rev. D 96 (2017) 045009 [arXiv:1703.05563] [INSPIRE].

[19] D. Anselmi and M. Piva, Quantum gravity, fakeons and microcausality, JHEP 11 (2018) 021 [arXiv: 1806.03605] [INSPIRE].

[20] D. Anselmi and A. Marino, Fakeons and microcausality: light cones, gravitational waves and the Hubble constant, Class. Quant. Grav. 37 (2020) 095003 [arXiv:1909.12873] [INSPIRE].

[21] I. Antoniadis, E. Dudas and D.M. Ghilencea, Supersymmetric models with higher dimensional operators, JHEP 03 (2008) 045 [arXiv:0708.0383] [INSPIRE]. 
[22] E. Dudas and D.M. Ghilencea, Effective operators in SUSY, superfield constraints and searches for a UV completion, JHEP 06 (2015) 124 [arXiv: 1503.08319] [INSPIRE].

[23] T. Fujimori, M. Nitta and Y. Yamada, Ghostbusters in higher derivative supersymmetric theories: who is afraid of propagating auxiliary fields?, JHEP 09 (2016) 106 [arXiv: 1608.01843] [INSPIRE].

[24] T. Fujimori, M. Nitta, K. Ohashi and Y. Yamada, Ghostbusters in $f(R)$ supergravity, JHEP 05 (2018) 102 [arXiv:1712.05017] [INSPIRE].

[25] J.F. Donoghue and G. Menezes, Ostrogradsky instability can be overcome by quantum physics, Phys. Rev. D 104 (2021) 045010 [arXiv:2105.00898] [INSPIRE].

[26] F. Arici, D. Becker, C. Ripken, F. Saueressig and W.D. van Suijlekom, Reflection positivity in higher derivative scalar theories, J. Math. Phys. 59 (2018) 082302 [arXiv:1712.04308] [INSPIRE].

[27] J. Gomis and S. Weinberg, Are nonrenormalizable gauge theories renormalizable?, Nucl. Phys. B 469 (1996) 473 [hep-th/9510087] [INSPIRE].

[28] D. Anselmi, Renormalization of gauge theories without cohomology, Eur. Phys. J. C 73 (2013) 2508 [arXiv: 1301.7577] [INSPIRE].

[29] A. Quadri, Background field method and generalized field redefinitions in effective field theories, Eur. Phys. J. Plus 136 (2021) 695 [arXiv:2102.10656] [INSPIRE].

[30] G.H. Derrick, Comments on nonlinear wave equations as models for elementary particles, J. Math. Phys. 5 (1964) 1252 [inSPIRE].

[31] J. Gasser and H. Leutwyler, Chiral perturbation theory to one loop, Annals Phys. 158 (1984) 142 [INSPIRE].

[32] J. Bijnens, G. Colangelo and G. Ecker, The mesonic chiral Lagrangian of order $p^{6}$, JHEP 02 (1999) 020 [hep-ph/9902437] [INSPIRE].

[33] J. Bijnens, N. Hermansson-Truedsson and S. Wang, The order $p^{8}$ mesonic chiral Lagrangian, JHEP 01 (2019) 102 [arXiv:1810.06834] [INSPIRE].

[34] L. Graf, B. Henning, X. Lu, T. Melia and H. Murayama, 2, 12, 117, 1959, 45171, 1170086 , ...: a Hilbert series for the QCD chiral Lagrangian, JHEP 01 (2021) 142 [arXiv: 2009.01239] [INSPIRE].

[35] H. Motohashi and T. Suyama, Third order equations of motion and the Ostrogradsky instability, Phys. Rev. D 91 (2015) 085009 [arXiv:1411.3721] [INSPIRE].

[36] H. Motohashi, K. Noui, T. Suyama, M. Yamaguchi and D. Langlois, Healthy degenerate theories with higher derivatives, JCAP 07 (2016) 033 [arXiv: 1603.09355] [INSPIRE].

[37] M. Crisostomi, R. Klein and D. Roest, Higher derivative field theories: degeneracy conditions and classes, JHEP 06 (2017) 124 [arXiv: 1703.01623] [INSPIRE].

[38] H. Motohashi, T. Suyama and M. Yamaguchi, Ghost-free theory with third-order time derivatives, J. Phys. Soc. Jap. 87 (2018) 063401 [arXiv:1711.08125] [InSPIRE].

[39] H. Motohashi, T. Suyama and M. Yamaguchi, Ghost-free theories with arbitrary higher-order time derivatives, JHEP 06 (2018) 133 [arXiv:1804.07990] [INSPIRE].

[40] A. Ganz and K. Noui, Reconsidering the Ostrogradsky theorem: higher-derivatives Lagrangians, ghosts and degeneracy, Class. Quant. Grav. 38 (2021) 075005 [arXiv: 2007.01063] [INSPIRE].

[41] C. Grosse-Knetter, Effective Lagrangians with higher derivatives and equations of motion, Phys. Rev. D 49 (1994) 6709 [hep-ph/9306321] [InSPIRE]. 